TRANSACTIONS OF THE

AMERICAN MATHEMATICAL SOCIETY

Volume 350, Number 12, December 1998, Pages 4973-4991

S $0002-9947(98) 02114-\mathrm{X}$

\title{
AN INDEX FOR PERIODIC ORBITS OF LOCAL SEMIDYNAMICAL SYSTEMS
}

\author{
CHRISTIAN C. FENSKE
}

\begin{abstract}
We define an index of Fuller type counting the number of periodic orbits of a semiflow on an ANR by a suitable approximation process.
\end{abstract}

\section{INTRODUCTION}

In this article it is our aim to define an index counting the periodic orbits of a semiflow on an ANR. Historically, the first definition of an index counting the periodic orbits of a smooth vectorfield on a finite dimensional manifold was given by Fuller [Fu]. Fuller's article already contains all relevant ideas for the analytical as well as the topological approach to this problem. A purely analytical treatment via bifurcation theory was given by Chow and Mallet-Paret [CMP]. In their article they also indicated a way to generalize the index to an infinite dimensional situation, viz., periodic solutions of functional differential equations. (To the present author's knowledge many readers of [CMP] have been puzzled by the question of why it is sufficient in this paper to restrict the argument to period-doubling bifurcations. The answer can be found in an article by Dancer and Toland [DT] in Theorem 2.8. I am very grateful to J.F. Toland for pointing this out to me.)

In [F2] the author presented a topological approach. For later purposes we sketch some details.

Definition 1. A local semiflow on a topological space $X$ consists of an open set $\mathcal{D} \subset X \times[0, \infty)$ and a continuous map $\Phi: \mathcal{D} \rightarrow X$ such that $\left(\right.$ with $\left.\phi_{t} x=\Phi(x, t)\right)$

i) $X \times\{0\} \subset \mathcal{D}$.

ii) For each $x \in X$ there is an $\omega_{x} \in(0, \infty]$ such that $(x, t) \in \mathcal{D}$ iff $0 \leq t<\omega_{x}$.

iii) $\phi_{0} x=x$ for $x \in X$.

iv) If $(x, t) \in \mathcal{D}$ and $\left(\phi_{t} x, s\right) \in \mathcal{D}$, then $(x, t+s) \in \mathcal{D}$ and $\phi_{t+s} x=\phi_{t} \circ \phi_{s} x$.

When $\Phi$ is given, we will find it convenient to write $\mathcal{D}(\Phi):=\mathcal{D}$. Let $x \in X$ and $\phi_{t} x=x$ for some $t>0$. Then $\omega_{x}=\infty, x$ is a periodic point, and $t$ is a period of $x$. If $\phi_{t} x=x$ for all $t \geq 0$, then $x$ is a rest point; otherwise there is a minimal $p>0$ with $\phi_{p} x=x$ and $t$ is a multiple of $p$. Then $m(x, t):=t / p$ is called the multiplicity of $(x, t)$ and $p(x):=p$ the minimal period. If $\gamma$ is the orbit of $x$, we write $p(\gamma):=p(x)$ and $m(\gamma, t):=t / p(\gamma)$.

Received by the editors November 20, 1995 and, in revised form, November 19, 1996.

1991 Mathematics Subject Classification. Primary 54H20; Secondary 58F25, 58F32, 55M25.

Key words and phrases. Index, periodic orbit, semiflow, Fuller index, functional differential equation. 
The simplest case occurs when $X$ is open in $\mathbf{R}^{n}, U$ is open in $X \times[0, \infty)$ with $\operatorname{cl} U \subset \mathcal{D}(\Phi)$ and $P:=P(\Phi):=\left\{(x, t) \in \operatorname{cl} U \mid \phi_{t}=x\right\}$ is a compact subset of $U$. Call $\tau \in H^{n}(X \times X, X \times X \backslash \Delta)$ the orientation and define $g: U \rightarrow X \times X$ by $g(x, t):=\left(x, \phi_{t} x\right)$. Then the homological index $I(X, \Phi, U)$ is defined to be the image of $\tau$ under

$$
\bar{H}^{n}(X \times X, X \times X \backslash \Delta) \stackrel{g^{*}}{\longrightarrow} \bar{H}^{n}(U, U \backslash P) \cong H_{1}^{c} P \stackrel{i_{*}}{\longrightarrow} H_{1} U
$$

Here, $\bar{H}$ is Alexander-Spanier cohomology, $\cong$ is Poincaré duality(cf. [M], p. 363), and $i: P \rightarrow U$ is the inclusion. In [F2] it is explained how one may generalize this result to the case where $X$ is an ANR (cf. $[\mathrm{H}]$ for facts about ANR), $\Phi$ has a compact attractor, and $\phi_{t_{0}}$ is locally compact for some $t_{0}>0$. A reader who likes fancy constructions may wish to describe $I(X, \Phi, U)$ in terms of Dold's transfer; this is spelt out in $[\mathrm{Sr} 1]$.

The definition of $I(X, \Phi, U)$ suffers from a major drawback: namely if $H_{1} U=0$, then the index will always be trivial. In [F3] we explained how to circumvent this difficulty in the case of a functional differential equation with a $C^{2}$ right hand side. In this situation, one can define a numerical index $i(X, \Phi, U) \in \mathbf{Q}$ due to the fact that for functional differential equations a Kupka-Smale type result is available. Assuming the right hand side to be $C^{2}$ (in an infinite-dimensional Banach space), however, rules out the most important applications in the case of (local semiflows generated by) functional differential equations or parabolic semilinear equations, since differentiability is a severe restriction in infinite dimensions. A first attempt to circumvent this difficulty was undertaken by A.J.B. Potter who in $[\mathrm{P} 1],[\mathrm{P} 2]$ considered a class of semiflows in Banach spaces that are approximable by $C^{2}-$ flows on finite-dimensional subspaces. This class contains many important cases of functional-differential equations, but his definition of the Fuller index was not completely satisfactory inasmuch as the index was not defined to be a rational number but rather a sequence of rational numbers (due to the fact that it is not clear that the indices of the approximating semiflows stabilize). In the present article we intend to define a numerical index for periodic orbits on ANR without any assumptions on differentiability. The idea is that the above definition of $I(X, \Phi, U)$ works for arbitrary mappings which need not be a local semiflow. Note, however, that although the index may be defined for "parametrized" mappings $F: \operatorname{cl} U \rightarrow X$, the index will enjoy the properties listed below only in the case of local semiflows. Cf. [Sr2], where it is shown that for any isolated component $C$ of periodic orbits of the Seifert flow on $S^{3}$ there is an arbitrarily small perturbation of the Seifert flow (as a parametrized mapping) which has no periodic point near $C$. Since the Fuller index of $C$ is easily computed to be nonzero, this would contradict the properties of additivity and homotopy invariance if these properties could be extended to the case of parametrized mappings. This shows that one has to exercise extreme care when using a parametrized mapping in approximating a local semiflow. Our idea here is to approximate $\Phi$ by a mapping $F: \operatorname{cl} U \rightarrow X$ such that $P(F):=$ $\{(x, t) \in \operatorname{cl} U \mid F(x, t)=t\}$ consists of finitely many one-spheres which correspond in a natural way to periodic orbits of $\Phi$ plus other components each of which is contained in a contractible set. This approach, however, needs some care: A brute force approach would approximate $\Phi$ by a smooth finite dimensional mapping $\Psi$ and then look for a regular value $y$ of $\operatorname{pr}_{X}-\Psi$ near 0 . Then, of course, one would have that $\{(x, t) \mid \Psi(x, t)=x-y\}$ consists of finitely many (homotopy-) 
one-spheres. These, however, need not have any relation to the original periodic orbits, and there is no conceivable way to assign an orientation or a multiplicity to these spheres. For example, one can imagine a semiflow $\Phi$ with $P(\Phi)=S^{1} \times S^{1}$ such that the flowlines are parallel to the first factor, i.e., $S^{1} \times\{a\}$ with $a \in S^{1}$. But for the approximating $F, P(F)$ might well look like $\bigcup_{i=1}^{n}\left\{b_{i}\right\} \times S^{1}$. So we see that in constructing the approximation we should retain as much structure as possible of the original periodic orbits. Of course, one will have the impression that our construction is unnecessarily complicated (though in a subsequent paper we intend to give an axiomatic description for the Fuller index) and there remains the challenge to find a more conceptual approach to the definition of an index for periodic orbits. Since the circle group $S^{1}$ acts on $P(\Phi)$, it would be tempting to look for an equivariant approximation, but it seems that this will not work since the action of $S^{1}$ cannot be extended over a neighbourhood of $P(\Phi)$. There is, however, in $[\mathrm{GN}]$ an extremely interesting approach using algebraic $K$-theory and Hochschild homology. Although in [GN] this approach is limited to the case of smooth flows on closed oriented manifolds, it seems possible that it may be generalized to the present situation.

\section{The SET-UP}

We shall deal with the following situation: $X$ is an ANR, $\Omega \subset X \times[0, \infty)$ is open, $\Phi$ is a local semiflow on $X$ such that $\operatorname{cl} \Omega \subset \mathcal{D}(\Phi)$ and such that $\operatorname{cl} \Phi(\Omega)$ is compact. We call $P(\Phi, \Omega):=\left\{(x, t) \in \operatorname{cl} \Omega \mid \phi_{t} x=x\right\}$, and we assume that $P(\Phi, \Omega)$ is a compact subset of $\Omega$. (In a later article we shall explain how to replace the compactness condition on $\mathrm{cl} \Phi(\Omega)$ by the assumption that $\Phi$ possesses a compact attractor plus some local compactness assumption.)

We say that an index is defined for triples $(X, \Phi, \Omega)$ satisfying the above conditions if to each such triple we can associate a rational number $i(X, \Phi, \Omega)$ such that:

(A) The index is additive, i.e.,

$$
i(X, \Phi, \Omega)=i\left(X, \Phi, \Omega_{1}\right)+i\left(X, \Phi, \Omega_{2}\right)
$$

if $\Omega$ is the disjoint union of open sets $\Omega_{1}$ and $\Omega_{2}$.

(H) The index is homotopy invariant: Assume that $\Omega \subset X \times[0, \infty) \times[0,1]$ is open and that we have a continuous mapping

$$
\begin{aligned}
& \Xi: \operatorname{cl} \Omega \rightarrow X, \\
& (x, t, \alpha) \mapsto \Xi^{\alpha}(x, t)
\end{aligned}
$$

where for each $\alpha \in[0,1] \quad \Xi^{\alpha}$ is a local semiflow with $\operatorname{cl} \Omega^{\alpha} \subset \mathcal{D}\left(\Xi^{\alpha}\right)$ where $\Omega^{\alpha}=\Omega \cap(X \times[0, \infty) \times\{\alpha\}), \Xi(\operatorname{cl} \Omega)$ is a compact set and $\{(x, t, \alpha) \in$ $\operatorname{cl} \Omega \mid \Xi(x, t, \alpha)=x\}$ is a compact subset of $\Omega$. Then

$$
i\left(X, \Xi^{0}, \Omega^{0}\right)=i\left(X, \Xi^{1}, \Omega^{1}\right) .
$$

(N) The index is normalized: $i(X, \Phi, \Omega)=i / m$, if $P$ consists of a single periodic orbit $\gamma$ of multiplicity $m$ and $i$ is the fixed point index of the Poincaré mapping associated with $\gamma$ (cf. [F1] for the definition of the Poincaré mapping for continuous semiflows on ANR). Equivalently, we could demand that $I(X, \Phi, U)=i(X, \Phi, U)[\gamma]$ where $[\gamma]$ is the homology class in $H_{1} U$ represented by the map $\tau \mapsto \phi_{t \tau} x$ on $[0,1]$ if $(x, t)$ is a point on $\gamma, t=m p(x)$, and $U$ is a 
neighbourhood of $\Phi(\{x\} \times[0, t]) \times\{t\}$ such that $[\gamma]$ represents a generator of $H_{1} U$.

\section{The MAIn Result}

Theorem. An index is defined for triples $(X, \Phi, \Omega)$ where $X$ is an ANR, $\Omega \subset$ $X \times[0, \infty)$ is open, and $\Phi$ is a local semiflow on $X$ such that $\operatorname{cl} \Omega \subset \mathcal{D}(\Phi), \operatorname{cl} \Phi(\Omega)$ is compact and $P:=P(\Phi, \Omega)$ is a compact subset of $\Omega$.

Proof. The proof consists of three main parts: First, we will "approximate" $\Phi$ by a mapping on a suitable nerve (cf. $[\mathrm{H}]$ for definitions and facts about nerves, realizations, and canonical projections) and define a numerical index for the approximating map. The second task will then be to show that this index depends neither on the choice of nerve nor on the particular approximation. Finally, we will have to verify properties $(A),(H)$, and $(\mathrm{N})$.

Part 1. We start by several reductions:

1.1. Since $P$ is a compact subset of $\Omega$, there are no rest points in $\Omega$, so $\operatorname{pr}_{2} P$ is a compact subset of $(0, \infty)$. So we may replace $\Omega$ by a neighbourhood of $P$ such that $\operatorname{pr}_{2} \operatorname{cl} \Omega$ is a compact subset of $(0, \infty)$. Let $t_{-}=\inf \operatorname{pr}_{2} \operatorname{cl} \Omega$ and $t_{+}=\operatorname{sup~pr}_{2} \operatorname{cl} \Omega$. Let $p_{-}:=\inf \left\{p(x) \mid x \in \operatorname{pr}_{1} P\right\}$ and $M:=\left[t_{+} / p_{-}\right]$, so $M$ is the greatest multiplicity which can occur for a periodic orbit in $\Omega$.

1.2. For each periodic point $(x, t)$ of $\Phi$ in $\Omega$ choose a tube $T_{\gamma}$ around $\gamma:=$ $\Phi(\{x\} \times[0, p(x)])$. (In [F1] it is shown that for a periodic point $x$ and neighbourhood $U$ of $\gamma$ in $X$ there are a neighbourhood $V \subset U$ of $\gamma$ and a set $S$ containing $x$ such that for each $y \in \Sigma:=S \cap V$ the map $\tau$ given by $\tau(y):=\inf \left\{s>0 \mid \phi_{s} y \in S\right\}$ is defined and continuous and such that $\Phi\left(\operatorname{cl} V \times\left[0, t_{+}\right]\right) \subset U$.) We then call $T_{\gamma}:=V$ a tube around $\gamma$ and $S$ a section at $x$. Since we may choose an open set $\Omega^{\prime}$ with $P(\Phi) \subset \Omega^{\prime} \subset \operatorname{cl} \Omega^{\prime} \subset \Omega$ and replace $\operatorname{cl} \Omega$ by $\operatorname{pr}_{1}^{-1}\left(\bigcup_{\gamma} T_{\gamma}\right) \cap \operatorname{cl} \Omega^{\prime}$, we may assume that, for each $(x, t) \in \operatorname{cl} \Omega, x$ is contained in a tube of a periodic point in $P(\Phi)$. We let $\tau_{1}:=\tau$ and $\tau_{m}(y):=\tau\left(\Phi\left(y, \tau_{m-1}(y)\right)\right)$ as long as this is defined.

1.3. Choose a compact ANR $Y$ with $\operatorname{cl} \Phi(\Omega) \subset Y$ and let $U=(Y \times[0, \infty)) \cap \Omega$. This is achieved as follows: Embed $X$ as a closed subset in a normed linear space $E$. Then let $\mathcal{O}$ be a neighbourhood of $X$ in $E$ such that there is a retraction $r: \mathcal{O} \rightarrow X$. By a result of $[\mathrm{G}]$ there is a compact ANR $C$ such that $\operatorname{cl} \Phi(\Omega) \subset C \subset \mathcal{O}$. Let $Y:=r(C)$.

1.4. Since we want the index to be additive, we may assume that $Y$ is connected. (Obviously, we may replace $Y$ by its connected components which are again ANR.)

Part 2: Definition of the index. The main tool in the proof will be the concept of convenient approximation: Before we introduce this concept, we fix some notation. If $\alpha$ is a (locally finite) open cover of a space $X$, we denote by $N_{\alpha}$ the nerve of $\alpha$ and, for $U \in \alpha$, by $\langle U\rangle_{\alpha}$ the vertex corresponding to $U$. Similarly, if $U_{1}, \ldots, U_{n} \in \alpha$ and $\bigcap_{i=1}^{n} U_{i} \neq \emptyset$, then $\left\langle U_{1}, \ldots, U_{n}\right\rangle_{\alpha}$ denotes the simplex with vertices $\left\langle U_{1}\right\rangle_{\alpha}, \ldots,\left\langle U_{n}\right\rangle_{\alpha}$. By "simplex" we always mean "closed simplex" and by st we denote the open star.

The idea in the proof is to construct a suitable covering $\alpha$ of $Y$ by first covering (a neighbourhood of) the periodic orbits of $\Phi$ by "tubes" and then the complement 
of this neighbourhood. Moreover, we include some "redundant" sets in the covering (namely, we choose an additional set in each maximal intersection) which will provide us with enough space to perform our approximation. Then we choose a canonical projection $p_{\alpha}: Y \rightarrow N_{\alpha}$ which maps some of the periodic orbits homeomorphically into the one-skeleton of $N_{\alpha}$ and we choose a realization $i_{\alpha}: N_{\alpha} \rightarrow Y$ which is inverse to $p_{\alpha}$ on this part of the one-skeleton. (A "realization" of $N_{\alpha}$ is just a continuous mapping $N_{\alpha} \rightarrow Y$. In ANR-spaces, one has control over the size of the images of the simplices of $N_{\alpha}$ under $i_{\alpha}$ (cf. [H]).) A convenient approximation will then be an approximation to the mapping given by $(x, t) \mapsto p_{\alpha} \phi_{t} i_{\alpha} x$ such that the fixed point set $\left\{(x, t) \mid f_{\alpha}(x, t)=x\right\}$ consists of the part of the one-skeleton corresponding to the periodic orbits plus some components (the projection onto $N_{\alpha}$ of) each of which is contained in a contractible subset of $N_{\alpha}$. So we can hope that these do not contribute to the index. The approximation itself is done by moving points which are not on the part of the one-skeleton corresponding to the periodic orbits into the direction of the vertices corresponding to the redundant sets of the covering. The details are, however, fairly laborious and will be relegated to the appendix.

Definition 2. Let $\Phi$ be as in Part 1. A convenient approximation for $\Phi$ consists of

- a finite open cover $\alpha$ of $Y$ with nerve $N_{\alpha}$, canonical projection $p_{\alpha}: Y \rightarrow N_{\alpha}$, and realization $i_{\alpha}: N_{\alpha} \rightarrow Y$ such that $I(Y, \Phi, U)$ is defined and does not depend on the choices involved in the definition (cf. [F2]) and

- a continuous mapping $f_{\alpha}: \operatorname{cl}_{\alpha}^{-1}(U) \rightarrow N_{\alpha}$ which is homotopic to $p_{\alpha} \Phi j_{\alpha}$ on $\mathrm{cl}_{\alpha}^{-1}(U)$ (where here and in what follows we write $j_{\alpha}(x, t):=\left(i_{\alpha} x, t\right)$ for $x \in N_{\alpha}$ and $\left.t \in \mathbf{R}\right)$ via a homotopy $H$ such that $H(x, t) \neq x$ whenever $\left(i_{\alpha} x, t\right) \in \partial U$ and such that, for $(x, t) \in j_{\alpha}^{-1}(U), f_{\alpha}(x, t) \in \operatorname{st} \sigma$ if $\sigma$ is the minimal simplex in $N_{\alpha}$ with $p_{\alpha} \phi_{t} i_{\alpha} x \in \sigma$.

Moreover, we require that the following conditions hold if we fix a euclidean metric $d$ on $N_{\alpha}$ :

1) a) $\alpha$ decomposes as $\alpha=\alpha_{0} \cup \alpha^{*}$ where $\alpha_{0}$ is a covering of $Y$ and for each maximal simplex $\sigma$ of $A_{0}:=N_{\alpha_{0}}$ there is a simplex of $N_{\alpha}$ consisting of $\sigma$ and a vertex $\left\langle U_{\sigma}\right\rangle_{\alpha}$ with $U_{\sigma} \in \alpha^{*}$ such that the following holds: if $\sigma_{1}, \ldots, \sigma_{m}$ are maximal simplices of $A_{0}$, then $\left\langle U_{\sigma_{1}}\right\rangle_{\alpha}, \ldots,\left\langle U_{\sigma_{m}}\right\rangle_{\alpha}$ form a simplex iff there is a maximal simplex $\sigma$ in $A_{0}$ such that each $\sigma_{j}$ meets $\sigma$.

b) If $\sigma_{1}, \ldots, \sigma_{m}$, and $\tau$ are maximal simplices in $A_{0}$ and $\tau^{\prime}$ is a face of $\tau$ such that $\left\langle U_{\sigma_{1}}\right\rangle, \ldots,\left\langle U_{\sigma_{m}}\right\rangle$ and $\tau^{\prime}$ form a simplex, then $\left\langle U_{\sigma_{1}}\right\rangle, \ldots,\left\langle U_{\sigma_{m}}\right\rangle$ and $\tau$ form a simplex. Moreover, if $E^{\prime}$ is the set of vertices of $\tau^{\prime}$ and $E$ is the set of vertices of $\tau$, then $\bigcap_{i=1}^{m} U_{\sigma_{i}} \cap \bigcap_{\langle V\rangle \in E^{\prime}} V=\bigcap_{i=1}^{m} U_{\sigma_{i}} \cap \bigcap_{\langle V\rangle \in E} V$.

c) If $\sigma$ is a simplex in $N_{\alpha}$ containing a vertex $\langle W\rangle_{\alpha}$ corresponding to $W \in \alpha^{*}$ such that $\sigma \cap A_{0}$ is either empty or a proper face in $A_{0}$, then $\sigma \cap$ st $\langle W\rangle_{\alpha}$ does not belong to the range of $p_{\alpha}$.

2) There are finitely many periodic orbits $\gamma_{1}, \ldots, \gamma_{s}$ of $\Phi$ and a decomposition $\alpha_{0}=\alpha_{1} \cup \alpha_{2}$ such that $\alpha_{1}$ decomposes into tubes around $\gamma_{1}, \ldots, \gamma_{s}$ which cover $\operatorname{pr}_{1} P$ and such that the closure of each element in $\alpha_{2}$ is disjoint from $\operatorname{pr}_{1} P$ and such that, for $j=1, \ldots, s$, the following holds: if $\Gamma_{j}$ denotes the union of all $W \in \alpha_{1}$ which meet $\gamma_{j}$, then for a periodic point $x \in \Gamma_{j}$ we have that $\left|p(x)-k p\left(\gamma_{j}\right)\right|<p_{-} /(2 M)$ for some positive integer $k \leq M$. Moreover, $\gamma_{1}, \ldots, \gamma_{s}$ are mapped homeomorphically by $p_{\alpha}$ into the 1-skeleton of $A_{0}$ with $i_{\alpha}$ being the 
inverse of $p_{\alpha} \mid \bigcup_{i=1}^{s} \gamma_{i}$ on $\gamma_{i}^{\prime}=p_{\alpha}\left(\gamma_{i}\right)$. Finally, $f_{\alpha}(x, t)=p_{\alpha} \Phi\left(i_{\alpha} x, t\right)$ if $x \in \gamma_{j}^{\prime}$ for some $j=1, \ldots, s$ and $t$ is a period of $x$. We call $\gamma_{1}, \ldots, \gamma_{s}$ (or $\gamma_{1}^{\prime}, \ldots, \gamma_{s}^{\prime}$ ) the distinguished orbits of $f_{\alpha}$.

3) Let $P_{\alpha}:=\left\{(x, t) \in j_{\alpha}^{-1}(U) \mid f_{\alpha}(x, t)=x\right\}$. For $j=1, \ldots, s$ let $M_{j}:=$ $\left\{t \mid \gamma_{j} \times\{t\} \subset P(\Phi)\right\}$. If $j \in\{1, \ldots, s\}$ and $t \in M_{j}$, then $P_{\alpha}$ contains $\gamma_{j}^{\prime} \times\{t\}$ as an isolated component, and the projection (onto $N_{\alpha}$ ) of each other component is contained in a contractible subset of $N_{\alpha}$. In particular, $\gamma_{1}^{\prime}, \ldots, \gamma_{s}^{\prime}$ are isolated in $\operatorname{pr}_{1} P_{\alpha}$.

4) Call $A_{*}$ the subcomplex of $N_{\alpha}$ containing all vertices $\langle W\rangle$ with $W \in \alpha^{*}$. Then there are neighbourhoods $W_{1}, \ldots, W_{s}$ of $\gamma_{1}^{\prime}, \ldots, \gamma_{s}^{\prime}$ in $N_{\alpha}$ and a $\delta>0$ such that $d\left(f_{\alpha}\left(x, t^{\prime}\right), A_{*}\right)<d\left(x, A_{*}\right)$ whenever $\left(x, t^{\prime}\right) \in \bigcup_{t \in M_{j}}\left[\operatorname{cl} W_{j} \times[t-\delta, t+\delta] \backslash\left(\gamma_{j}^{\prime} \times\{t\}\right)\right]$ for some $j=1, \ldots, s$.

5) If $\gamma \times\{t\}$ is isolated in $P(\Phi)$ and $V$ is an isolating neighbourhood of $\gamma^{\prime} \times\{t\}$ in $j_{\alpha}^{-1}(U)$ (where $\gamma^{\prime}=p_{\alpha}(\gamma)$ ), then $I\left(Y, \Phi, j_{\alpha}^{-1}(V)\right)=j_{\alpha *} I\left(N_{\alpha}, f_{\alpha}, V\right)$.

Observe that although it looks awkward, Condition 1) c) can easily be fulfilled if we can fulfill 1) a) and b). Namely, we will choose the canonical projection in a specific way as in ([Du], VIII.4 and 5): If $X$ is a paracompact space (in fact, a compact space would be sufficient for our purposes) and $\alpha$ is a locally finite open cover of $X$ (again a finite cover would suffice), choose a partition of unity, $\left(\psi_{U}\right)_{U \in \alpha}$ subordinated to $\alpha$ (i.e., for each $U \in \alpha$ the support of $\psi_{U}$ is nonempty and contained in $U$ ) and define $p_{\alpha}: X \rightarrow N_{\alpha}$ by $p_{\alpha}(x)=\sum_{U \in \alpha} \psi_{U}(x)\langle U\rangle_{\alpha}$. Consider then a simplex $\Sigma$ containing a vertex in $A_{*}$ and meeting $A_{0}$ in a simplex $\tau^{\prime}=\Sigma \cap A_{0}$ which is a proper face of a maximal simplex $\tau$ in $A_{0}$. Then let $y \in \Sigma \backslash \tau^{\prime}$. We claim that $y \notin p_{\alpha}(Y)$. Since $y \notin \tau^{\prime}$, there is a vertex $\left\langle U_{\sigma}\right\rangle$, say, of $\Sigma$ in $A_{*}$ such that $y \in$ st $\left\langle U_{\sigma}\right\rangle$. By Condition 1) b) $\left\langle U_{\sigma}\right\rangle,\left\langle U_{\tau}\right\rangle$, and $\tau$ will then form a simplex. Moreover, if $E^{\prime}$ denotes the set of vertices of $\tau^{\prime}$ and $E$ the set of vertices of $\tau$, we have that $U_{\sigma} \cap \bigcap_{\langle V\rangle \in E^{\prime}} V \subset \bigcap_{\langle V\rangle \in E} V$. So if there were an $x \in Y$ with $p_{\alpha}(x)=y$, we would have that $x \in U_{\sigma} \cap \bigcap_{\langle V\rangle \in E^{\prime}} V \subset \bigcap_{\langle V\rangle \in E} V$. But then $x$ would have positive barycentric coordinates with respect to the vertices of $\tau$ not in $\tau^{\prime}$. In addition, we observe that $\Sigma$ cannot be a maximal simplex: Let $\left\langle U_{\sigma_{1}}\right\rangle, \ldots,\left\langle U_{\sigma_{m}}\right\rangle$ be the vertices of $\Sigma$ in $A_{*}$. Then we claim that $\left\langle U_{\sigma_{1}}\right\rangle, \ldots,\left\langle U_{\sigma_{m}}\right\rangle$, and $\tau$ form a simplex. But this is obvious since $\bigcap_{i=1}^{m} U_{\sigma_{i}} \cap \bigcap_{\langle V\rangle \in E^{\prime}} V \neq \emptyset$ and hence by Property 1) b) $\bigcap_{i=1}^{m} U_{\sigma_{i}} \cap \bigcap_{\langle V\rangle \in E} V \neq \emptyset$. If $\Sigma$ is a simplex which does not meet $A_{0}$, it is trivial that $\Sigma$ cannot be maximal (since $\alpha_{0}$ covers $Y$ ) and that $\Sigma$ cannot meet the range of $p_{\alpha}$.

We shall prove in the appendix that there exists a convenient approximation, and we shall now define the index $i(Y, \Phi, U)$ in terms of a convenient approximation. Let $\gamma_{1}, \ldots, \gamma_{s}$ be as in 2) in the definition and choose neighbourhoods with disjoint closures, $V_{1}, \ldots, V_{s}$, of $\gamma_{1}^{\prime}, \ldots, \gamma_{s}^{\prime}$ in $N_{\alpha}$ and a positive $\delta<p_{-} / 2$ such that for $j \in\{1, \ldots, s\}$

- $\gamma_{j}^{\prime}$ is a deformation retract of $V_{j}$;

$-\operatorname{cl} V_{j} \times[t-\delta, t+\delta] \subset j_{\alpha}^{-1}(U)$ for $t \in M_{j}:=\left\{\tau \mid \gamma_{j} \times\{\tau\} \subset P(\Phi)\right\}$;

$-\operatorname{cl} V_{j} \subset \operatorname{st} \gamma_{j}^{\prime}$. Let $x \in \operatorname{cl} V_{j}$ and call $\sigma$ the minimal simplex in $\gamma_{j}^{\prime}$ with $x \in \operatorname{st} \sigma$; then $f_{\alpha}(x, \tau) \in$ st $\sigma$ for $|\tau-t| \leq \delta$. This is possible since $\Phi\left(\gamma_{j} \times \mathbf{R}\right)=\gamma_{j}$ and $f_{\alpha}(x, t)=p_{\alpha} \Phi\left(i_{\alpha} x, t\right)$ if $x \in \gamma_{j}^{\prime}$ and $t \in M_{j}$.

$-i_{\alpha}^{-1}\left(V_{j}\right) \subset \Gamma_{j}$. 


$$
\begin{aligned}
& -d\left(f_{\alpha}\left(x, t^{\prime}\right), A_{*}\right)<d\left(x, A_{*}\right) \text { whenever } \\
& \qquad\left(x, t^{\prime}\right) \in \bigcup_{t \in M_{j}}\left[\operatorname{cl} V_{j} \times[t-\delta, t+\delta] \backslash\left(\gamma_{j}^{\prime} \times\{t\}\right)\right] .
\end{aligned}
$$

Then let $j=1, \ldots, s$ and $t \in M_{j}$, define $m_{j}(t)=t / p\left(\gamma_{j}\right)$, and write the homological index as $I\left(N_{\alpha}, f_{\alpha}, V_{j} \times(t-\delta, t+\delta)\right)=c_{t}\left[\gamma_{j}^{\prime}\right]$. Then we define

$$
\operatorname{ind}_{\alpha}(Y, \Phi, U)=\sum_{j=1}^{s} \sum_{t \in M_{j}} \frac{c_{t}}{m_{j}(t)} .
$$

Part 3: The index does not depend on the choices involved in the definition.

3.1. We first consider the case where we have convenient approximations $f_{\alpha}$ and $f_{\alpha}^{\prime}$ for the same $p_{\alpha} \Phi j_{\alpha}$. We choose $\gamma_{1}, \ldots, \gamma_{s}$ as in 2) in the definition and neighbourhoods $V_{1}, \ldots, V_{s}$ of $\gamma_{1}^{\prime} \ldots, \gamma_{s}^{\prime}$ and a $\delta>0$ as above. We will now describe a homotopy between $f_{\alpha}$ and $f_{\alpha}^{\prime}$ on $\mathrm{cl} V_{j} \times[t-\delta, t+\delta]$ when $t \in M_{j}$. Then let $x \in \operatorname{cl} V_{j}$ and $\left|t-t^{\prime}\right| \leq \delta$. By assumption there is a minimal simplex $\sigma$ in $\gamma_{j}^{\prime}$ such that $x \in$ st $\sigma$ and $f_{\alpha}\left(x, t^{\prime}\right), f_{\alpha}^{\prime}\left(x, t^{\prime}\right) \in$ st $\sigma$. So either $f_{\alpha}\left(x, t^{\prime}\right)$ and $f_{\alpha}^{\prime}\left(x, t^{\prime}\right)$ belong to the same simplex or to different simplices in st $\sigma$. Then let $\sigma_{1}$ denote the maximal simplex containing $p:=f_{\alpha}\left(x, t^{\prime}\right)$ and $\sigma_{2}$ the maximal simplex containing $q:=f_{\alpha}^{\prime}\left(x, t^{\prime}\right)$. Then $\sigma_{1}$ and $\sigma_{2}$ have a maximal common face $\tau$ and this face contains $\sigma$ and $\left\langle U_{\sigma_{1}}, U_{\sigma_{2}}\right\rangle_{\alpha}$. We will then obtain the required homotopy by joining $f_{\alpha}\left(x, t^{\prime}\right)$ to $f_{\alpha}^{\prime}\left(x, t^{\prime}\right)$ by a "broken line": Let $\lambda \in[0,1]$. If $\sigma_{1}=\sigma_{2}$, we let $\ell(p, q ; \lambda)=(1-\lambda) p+\lambda q$. If $\sigma_{1} \neq \sigma_{2}$, we choose the unique point $z \in \tau$ such that $d:=d(p, z)+d(z, q)$ is minimal, and we abbreviate $d_{1}:=d(p, z)$ and $d_{2}:=d(q, z)$. We then define $\ell(p, q ; \lambda):=$ $\left(1-d \lambda / d_{1}\right) p+d \lambda / d_{1} \cdot z$ if $0 \leq \lambda \leq d_{1} / d$ and $\ell(p, q ; \lambda):=(1-\lambda) d / d_{2} \cdot z+\left(d \lambda-d_{1}\right) / d_{2} \cdot q$ if $d_{1} / d \leq \lambda \leq 1$. We then define $h: \operatorname{cl} V_{j} \times[t-\delta, t+\delta] \times[0,1] \rightarrow N_{\alpha}$ by $h\left(x, t^{\prime}, \lambda\right):=$ $\ell\left(f_{\alpha}\left(x, t^{\prime}\right), f_{\alpha}^{\prime}\left(x, t^{\prime}\right) ; \lambda\right)$. Since $d\left(f_{\alpha}\left(x, t^{\prime}\right), A_{*}\right)<d\left(x, A_{*}\right)$ and $d\left(f_{\alpha}^{\prime}\left(x, t^{\prime}\right), A_{*}\right)<$ $d\left(x, A_{*}\right)$ for $\left(x, t^{\prime}\right) \in \partial\left(V_{j} \times[t-\delta, t+\delta]\right)$ and since the common face $\tau$ of $\sigma_{1}$ and $\sigma_{2}$ contains points in $A_{*}$, we will also have that $d\left(h\left(x, t^{\prime}, \lambda\right), A_{*}\right)<d\left(x, A_{*}\right)$ and consequently that $h\left(x, t^{\prime}, \lambda\right) \neq x$ for $\left(x, t^{\prime}\right) \in \partial\left(V_{j} \times[t-\delta, t+\delta]\right)$. So we have that $I\left(N_{\alpha}, f_{\alpha}, V_{j} \times(t-\delta, t+\delta)\right)=I\left(N_{\alpha}, f_{\alpha}^{\prime}, V_{j} \times(t-\delta, t+\delta)\right)$ due to the homotopy invariance of the homological index. (A priori, we only have that both indices have the same image in $H_{1}\left(V_{j} \times(t-\delta, t+\delta) \times[0,1]\right)$, but since the inclusions $\left(x, t^{\prime}\right) \mapsto\left(x, t^{\prime}, 0\right)$ and $\left(x, t^{\prime}\right) \mapsto\left(x, t^{\prime}, 1\right)$ induce isomorphisms this shows what we need.)

3.2. Next we consider the case where we have different realizations $i_{\alpha}, i_{\alpha}^{\prime}: N_{\alpha} \rightarrow Y$ and canonical projections: $p_{\alpha}, p_{\alpha}^{\prime}: Y \rightarrow N_{\alpha}$. But $p_{\alpha} \Phi j_{\alpha}$ and $p_{\alpha}^{\prime} \Phi j_{\alpha}^{\prime}$ are homotopic without fixed points on the boundary of $\mathrm{cl}_{\alpha}^{-1}(U)$ and by our assumption $p_{\alpha}$ and $p_{\alpha}^{\prime}$ have to coincide on $\gamma_{1}, \ldots, \gamma_{s}$ and $i_{\alpha}$ and $i_{\alpha}^{\prime}$ have to coincide on $\gamma_{1}^{\prime}, \ldots, \gamma_{s}^{\prime}$ as well. Since $p_{\alpha} \phi_{t} i_{\alpha} x=x$ for $x \in \gamma_{j}^{\prime}$ and $t \in M_{j}$, we may argue as in 3.1.

3.3. We now assume that there is a subcomplex $K$ of $N_{\alpha}$ such that $p_{\alpha}(Y) \subset K$. Call $\beta$ the covering $\{U \in \alpha \mid\langle U\rangle \in K\}$. We then must have $A_{0} \subset N_{\beta}$. We let $\beta_{0}:=\beta \cap \alpha_{0}$ and $\beta^{*}:=\beta \cap \alpha^{*}$. We use $p_{\alpha}$ as a canonical projection $p_{\alpha}: Y \rightarrow N_{\beta}$ and $i_{\beta}:=i_{\alpha} \mid N_{\beta}$. Then let $f_{\beta}$ be a convenient approximation. Choose a neighbourhood $\mathcal{O}$ of $N_{\beta}$ in $N_{\alpha}$ and a deformation retraction $r: \mathcal{O} \rightarrow N_{\beta}$ and define $f_{\alpha}(x, t):=f_{\beta}(r x, t)$ if $(r x, t) \in j_{\beta}^{-1}(\mathrm{cl} U)$. Obviously, $f_{\alpha}$ will be a convenient approximation. We then choose neighbourhoods $V_{1}, \ldots, V_{s}$ and a $\delta$ as in the definition of $\operatorname{ind}_{\beta}(Y, \Phi, U)$. 
By continuity there are neighbourhoods $\tilde{V}_{1}, \ldots, \tilde{V}_{s}$ with $\tilde{V}_{j} \subset r^{-1}\left(V_{j}\right)$ such that $d\left(f_{\alpha}\left(x, t^{\prime}\right), A_{*}\right)<d\left(x, A_{*}\right)$ whenever $\left(x, t^{\prime}\right) \in \partial\left(\tilde{V}_{j} \times[t-\delta, t+\delta]\right)$ for $t \in M_{j}$. By the property of weak commutativity (cf. [F2], Lemma 1) we have that

$$
\begin{aligned}
I\left(N_{\alpha}, f_{\alpha}, \tilde{V}_{j} \times[t-\delta, t+\delta]\right) & =j_{*} I\left(N_{\beta}, f_{\alpha}, V_{j} \times[t-\delta, t+\delta]\right) \\
& =j_{*} I\left(N_{\beta}, f_{\beta}, V_{j} \times[t-\delta, t+\delta]\right)
\end{aligned}
$$

(with $j: j_{\beta}^{-1}(U) \rightarrow j_{\alpha}^{-1}(U)$ being the inclusion), so $\operatorname{ind}_{\alpha}(Y, \Phi, U)=\operatorname{ind}_{\beta}(Y, \Phi, U)$.

3.4. Finally we have to deal with the case of two coverings $\alpha$ and $\beta$. If $\alpha=\alpha_{0} \cup \alpha^{*}$ and $\beta=\beta_{0} \cup \beta^{*}$ are finite open coverings as in Condition 1 of Definition 2, we write $\alpha_{0}=\alpha_{1} \cup \alpha_{2}$ and $\beta_{0}=\beta_{1} \cup \beta_{2}$ as in Condition 2 where $\alpha_{1}$ decomposes into tubes $T_{1}, \ldots, T_{r}$ around distinguished orbits $\gamma_{1}, \ldots, \gamma_{r}$ and, analogously, $\beta_{1}=T_{1}^{\prime} \cup \cdots \cup T_{s}^{\prime}$ with distinguished orbits $\gamma^{1}, \ldots, \gamma^{s}$. We will now form a covering $\kappa$ which refines both $\alpha$ and $\beta$ such that the set of distinguished orbits of $\kappa$ is $\left\{\gamma_{1}, \ldots, \gamma_{r}, \gamma^{1}, \ldots, \gamma^{s}\right\}$. Let these be numbered as $\gamma_{1}, \ldots, \gamma_{u}$ with $u \geq r$ (so $\gamma_{r+1}, \ldots, \gamma_{u}$ are simply those distinguished orbits $\gamma^{i}$ which are not contained among $\left.\gamma_{1}, \ldots, \gamma_{r}\right)$. We choose tubes $T_{1}^{\prime \prime}, \ldots, T_{u}^{\prime \prime}$ around $\gamma_{1}, \ldots, \gamma_{u}$ such that $T_{i}^{\prime \prime}=T_{i}$ if $i \leq r$ and $\gamma_{i} \notin\left\{\gamma^{1}, \ldots, \gamma^{s}\right\}$ and $T_{i}^{\prime \prime}=T_{i}^{\prime}$ if $r<i \leq u$, and, finally, $T_{i}^{\prime \prime}=T_{i} \cap T_{k}^{\prime}$ if $i \leq r$ and $\gamma_{i}=\gamma^{k}$. In the last case we intersect the elements of $\alpha_{1}$ and $\beta_{1}$ contained in $T_{i}$ and $T_{k}^{\prime}$, respectively, with $T_{i}^{\prime \prime}$, and we collect the elements of $T_{1}^{\prime \prime}, \ldots, T_{u}^{\prime \prime}$ into $\kappa_{1}$. We then let $\kappa_{2}$ consist of all nonempty intersections $V \cap V^{\prime}$ with $V \in \alpha_{2}$ and $V^{\prime} \in \beta_{0}$ or $V \in \alpha_{0}$ and $V^{\prime} \in \beta_{2}$. It is then obvious that $\kappa_{0}:=\kappa_{1} \cup \kappa_{2}$ refines $\alpha_{0}$ and $\beta_{0}$. If $\sigma=\left\langle U_{1}, \ldots, U_{n}\right\rangle$ is a maximal simplex of $N_{\kappa_{0}}$, we choose an open set $U_{\sigma}^{\prime} \subset \operatorname{cl} U_{\sigma}^{\prime} \subset \bigcap_{i=1}^{n} U_{i}$. If $\sigma_{0}$ is a maximal simplex of $N_{\kappa_{0}}$ and $\sigma_{1}, \ldots, \sigma_{m}$ are the maximal simplices which meet $\sigma_{0}$, then we let $U_{\sigma_{0}}:=\bigcup_{i=0}^{m} U_{\sigma_{i}}^{\prime}$. Call $\kappa^{*}$ the set of all $U_{\sigma}$ corresponding to maximal simplices of $N_{\kappa_{0}}$, and let $\kappa=\kappa_{0} \cup \kappa^{*}$. Choose a simplicial map $q_{\kappa \alpha}: N_{\kappa} \rightarrow N_{\alpha}$ by assigning to $\langle U\rangle$, if $U \in \kappa_{0}$, a vertex $\langle V\rangle$ where $U \subset V \in \alpha_{0}$. If $\sigma=\left\langle U_{1}, \ldots, U_{n}\right\rangle$ is a maximal simplex of $\alpha_{0}$, there is a maximal simplex $\tau$ of $N_{\kappa_{0}}$ containing the vertices $\left\langle U_{1}\right\rangle, \ldots,\left\langle U_{m}\right\rangle$. We then $\operatorname{map}\left\langle U_{\tau}\right\rangle$ to $\left\langle U_{\sigma}\right\rangle$. For the other vertices $\langle U\rangle$ corresponding to sets in $\kappa^{*}$ we choose an element $V \in \alpha_{0}$ with $U \subset V$ and let $q_{\kappa \alpha}\left(\langle U\rangle_{\kappa}\right)=\langle V\rangle_{\alpha}$. Moreover, we may use $q_{\kappa \alpha} \circ p_{\kappa}$ as a canonical projection for $N_{\alpha}$, so, by 3.3, we may assume that $q_{\kappa \alpha}$ is surjective. But this means, in particular, that we may assume that $q_{\kappa \alpha}$ maps the subcomplex containing the vertices corresponding to elements in $\kappa^{*}$ onto $A_{*}$. So (by slightly changing and simplifying the notation) we are reduced to the following situation: $\alpha=\alpha_{0} \cup \alpha^{*}$ and $\beta=\beta_{0} \cup \beta^{*}$ are coverings such that $\alpha$ refines $\beta, \alpha_{0}$ refines $\beta_{0}$, and the simplicial map $q_{\alpha \beta}: N_{\alpha} \rightarrow N_{\beta}$ is surjective and maps $A^{*}$ onto $B^{*}$ where $A^{*}\left(B^{*}\right)$ is the subcomplex of $N_{\alpha}\left(N_{\beta}\right)$ containing the vertices in $\alpha^{*}\left(\beta^{*}\right)$. Moreover, we may assume that the distinguished orbits $\gamma_{1}, \ldots, \gamma_{s}$ of $f_{\alpha}$ may be arranged as $\gamma_{1}, \ldots, \gamma_{r}, \gamma_{r+1}, \ldots, \gamma_{s}$ where $\gamma_{1}, \ldots, \gamma_{r}$ are the distinguished orbits of $f_{\beta}$. In order to distinguish between the $p_{\alpha} \gamma_{j}$ and the $p_{\beta} \gamma_{j}$ we let $\gamma_{j}^{\prime}:=p_{\alpha} \gamma_{j}$ for $j=1, \ldots, s$ and $\hat{\gamma}_{j}:=p_{\beta} \gamma_{j}$ for $j=1, \ldots, r$. We now view $N_{\alpha}$ and $N_{\beta}$ as subcomplexes of $N_{\alpha \cup \beta}$ and denote by $h: N_{\alpha \cup \beta} \times[0,1] \rightarrow N_{\alpha \cup \beta}$ the deformation retraction (cf. [F2]) such that $h(\cdot, 0)=\mathrm{id}$ and $h(\cdot, 1): N_{\alpha \cup \beta} \rightarrow N_{\beta}$ is a retraction such that $q_{\alpha \beta}:=h \mid N_{\alpha} \times\{1\}$.

Let $V_{1}, \ldots, V_{s}$ be neighbourhoods of $\gamma_{1}^{\prime}, \ldots, \gamma_{s}^{\prime}$ in $N_{\alpha}$ as in the definition of $\operatorname{ind}_{\alpha}(Y, \Phi, U)$. Then $V_{1}^{\prime}=q_{\alpha \beta}\left(V_{1}\right), \ldots, V_{s}^{\prime}=q_{\alpha \beta}\left(V_{s}\right)$ are open in $N_{\beta}$ and we may choose $V_{1}, \ldots, V_{r}$ so small that we may use $V_{1}^{\prime}, \ldots, V_{r}^{\prime}$ in the definition of $\operatorname{ind}_{\beta}(Y, \Phi, U)$. We now intend to invoke the property of homotopy invariance for 
the index $I(Y, \Phi, U)$ on the open subset $h\left(\bigcup_{i=1}^{r} V_{i} \times[0,1]\right)$ of $N_{\alpha \cup \beta}$. In the appendix describing the construction of a convenient approximation we will show how to construct an approximation (not necessarily a convenient one) $f_{\alpha \beta}$ to the map on $\operatorname{cl} j_{\alpha \cup \beta}^{-1}(U)$ which is given by $(h(x, \tau), t) \mapsto h\left(p_{\alpha} \phi_{t} i_{\alpha \cup \beta} h(x, \tau), \tau\right)$ for $(x, t) \in$ $\operatorname{cl} j_{\alpha}^{-1}(U)$ and $0 \leq \tau \leq 1$. Here and in what follows we are somewhat sloppy in our notation: of course, a point in $N_{\beta}$ need not be uniquely representable as $h(x, 1)$ with $x \in N_{\alpha}$ but we will always take care that all expressions are well-defined. Moreover, we shall construct $f_{\alpha \beta}$ in such a way that $f_{\alpha \beta} \mid \operatorname{cl} j_{\alpha}^{-1}(U)=: f_{\alpha}$ and $f_{\alpha \beta} \mid \operatorname{cl} j_{\beta}^{-1}(U)=$ : $f_{\beta}$ are convenient approximations to $p_{\alpha} \Phi j_{\alpha}$ and $p_{\beta} \Phi j_{\beta}$, respectively, such that $f_{\alpha \beta}(x, t) \neq x$ whenever $(x, t) \in \operatorname{cl} j_{\alpha \cup \beta}^{-1}(U)$ and $x \in \partial h\left(\bigcup_{i=1}^{s} V_{i} \times[0,1]\right)$. Call $N_{t}:=h\left(N_{\alpha} \times\{t\}\right)$. For $0 \leq t<1, N_{t}$ is homeomorphic to $N_{\alpha}$ and it has an obvious simplicial structure which is isomorphic to $N_{\alpha}$. Moreover, by our assumption, $N_{1}=N_{\beta}$. Our construction of $f_{\alpha \beta}$ will satisfy $f_{\alpha \beta}(h(x, \tau), t) \in N_{\tau}$ if $(h(x, \tau), t) \in j_{\alpha \cup \beta}^{-1}(U)$. For $0 \leq t \leq 1$ we denote by $i_{t}: N_{t} \rightarrow N_{\alpha \cup \beta}$ the inclusion and we define again $j_{t}$ by $j_{t}(x, s)=\left(i_{t} x, s\right)$.

We now arrange $\gamma_{1}, \ldots, \gamma_{r}, \gamma_{r+1}, \ldots, \gamma_{s}$ as follows: Choose $u \in\{0, \ldots, s-r\}$ such that, for $i \in\{1, \ldots, u\}, \gamma_{r+i}^{\prime}$ is mapped onto $\hat{\gamma}_{j}$ for some $j \in\{1, \ldots, r\}$ under $q_{\alpha \beta}$. Then there are three possible cases:

a) $j \leq r$ and no $\gamma_{r+i}^{\prime}(i \in\{1, \ldots, u\})$ is mapped onto $\hat{\gamma}_{j}$.

b) $j \leq r$ and $\gamma_{r+i}^{\prime}$ is mapped onto $\hat{\gamma}_{j}$ for some $i \in\{1, \ldots, u\}$.

c) $u<s-r$, so there is a $j>r+u$, and $\gamma_{j}^{\prime}$ is not mapped onto any of $\hat{\gamma}_{1}, \ldots, \hat{\gamma}_{r}$. Then let $j \in\{1, \ldots, s\}$ and $\gamma_{j} \times\{t\} \subset P(\Phi)$. Let $\tau \in[0,1]$. In cases a) and c) we let $W_{\tau}:=h\left(V_{j} \times\{\tau\}\right) \times(t-\delta, t+\delta)$. In case b) we may arrange our notation in such a way that (precisely) $\gamma_{r+1}^{\prime}, \ldots, \gamma_{r+v}^{\prime}$ (with $v \leq u$ ) are mapped onto $\hat{\gamma}_{j}$ by $q_{\alpha \beta}$. In this case we let $W_{\tau}=h\left(\left(V_{j} \cup \bigcup_{i=1}^{v} V_{r+i}\right) \times\{\tau\}\right) \times(t-\delta, t+\delta)$ and in each of these cases we let $W:=\bigcup_{\tau \in[0,1]} W_{\tau}$. If we choose $\epsilon>0$ small enough, we have that

$$
j_{1 *} I\left(N_{\beta}, f_{\beta}, W_{1}\right)=j_{1-\epsilon_{*}} I\left(N_{1-\epsilon}, f_{\alpha \beta}, W_{1-\epsilon}\right)
$$

by continuity of the topological index and the property of weak commutativity. Now we identify $\bigcup_{\tau \in[0,1-\epsilon]} W_{\tau}$ with $W_{0} \times[0,1-\epsilon]$ and we define a "homotopy"

$$
\begin{aligned}
H: & \bigcup_{\tau \in[0,1-\epsilon]} W_{\tau} \rightarrow N_{\alpha \cup \beta}, \\
\left(h(x, \tau), t^{\prime}\right) & \mapsto f_{\alpha \beta}\left(h(x, \tau), t^{\prime}\right) .
\end{aligned}
$$

By the homotopy invariance of the topological index this shows that

$$
j_{1-\epsilon_{*}} I\left(N_{1-\epsilon}, f_{\alpha \beta}, W_{1-\epsilon}\right)=j_{0 *} I\left(N_{\alpha}, f_{\alpha}, W_{0}\right)
$$

and so that

$$
j_{1_{*}} I\left(N_{\beta}, f_{\beta}, W_{1}\right)=j_{0 *} I\left(N_{\alpha}, f_{\alpha}, W_{0}\right) .
$$

We now have to relate the topological indices to the numerical indices. In case a) it is obvious that $\operatorname{ind}_{\alpha}\left(Y, \Phi, j_{\alpha}^{-1}\left(V_{j} \times(t-\delta, t+\delta)\right)\right)=\operatorname{ind}_{\beta}\left(Y, \Phi, j_{\beta}^{-1}\left(V_{j}^{\prime} \times(t-\delta, t+\delta)\right)\right)$. Similarly, in case c) we have that $\operatorname{ind}_{\alpha}\left(Y, \Phi, j_{\alpha}^{-1}\left(V_{j} \times(t-\delta, t+\delta)\right)\right)=0$ since $q_{\alpha \beta}$ maps $\gamma_{j}^{\prime}$ into the one-skeleton of $N_{\beta}$, so $q_{\alpha \beta} \gamma_{j}^{\prime}$ must contain points $x$ with $f_{\beta}(x, t) \neq x$ for all $t$ such that $\left(i_{\beta} x, t\right) \in U$. In case $\left.\mathrm{b}\right)$ we have to argue somewhat more carefully: For each $i \in\{1, \ldots, v\}$ there must be a $\tau_{r+i} \in(t-\delta, t+\delta)$ such that $f_{\alpha}\left(x, \tau_{r+i}\right)=x$ for $x$ on $\gamma_{r+i}^{\prime}$. Since for these $i$ we have that $q_{\alpha \beta} \gamma_{r+i}^{\prime}=\hat{\gamma}_{j}$, we must have that the 
$\gamma_{r+i}$ are contained in $\Gamma_{j}$. Then let $i \in\{1, \ldots, v\}$. We abbreviate $m:=m\left(\gamma_{j}, t\right)$, $m_{r+i}:=m\left(\gamma_{r+i}, \tau_{r+i}\right), p:=p\left(\gamma_{j}\right), p_{r+i}:=p\left(\gamma_{r+i}\right)$, and $V:=V_{j} \cup \bigcup_{i=1}^{v} V_{r+i}$. We now write

$$
\begin{gathered}
I\left(N_{\alpha}, f_{\alpha}, V \times(t-\delta, t+\delta)\right)=c_{j}\left[\gamma_{j}^{\prime}\right]+\sum_{i=1}^{v} c_{r+i}\left[\gamma_{r+i}^{\prime}\right] \text { and } \\
I\left(N_{\beta}, f_{\beta}, V_{j}^{\prime} \times(t-\delta, t+\delta)\right)=c\left[\hat{\gamma}_{j}\right]
\end{gathered}
$$

where for brevity we identify periodic orbits in $N_{\alpha}$ and $N_{\beta}$ with their images in $N_{\alpha \cup \beta}$. By our definition of $\operatorname{ind}_{\alpha}$ we have that

$$
\operatorname{ind}_{\alpha}\left(Y, \Phi, j_{\alpha}^{-1}(V \times(t-\delta, t+\delta))\right)=\frac{c_{j}}{m}+\sum_{i=1}^{v} \frac{c_{r+i}}{m_{r+i}}
$$

and

$$
\operatorname{ind}_{\beta}\left(Y, \Phi, j_{\beta}^{-1}\left(V_{j}^{\prime} \times(t-\delta, t+\delta)\right)\right)=\frac{c}{m} .
$$

So we have to show that

$$
\frac{c_{j}}{m}+\sum_{i=1}^{v} \frac{c_{r+i}}{m_{r+i}}=\frac{c}{m}
$$

Equality of the topological indices yields that (upon applying $q_{\alpha \beta_{*}}$ to $I\left(N_{\alpha}, f_{\alpha}, V \times\right.$ $(t-\delta, t+\delta)))$ :

$$
c_{j}\left[\hat{\gamma}_{j}\right]+\sum_{i=1}^{v} c_{r+i} q_{\alpha \beta_{*}}\left[\gamma_{r+i}^{\prime}\right]=c\left[\hat{\gamma}_{j}\right]
$$

Now we have that $\left|\tau_{r+i}-t\right|<\delta$. But $\tau_{r+i}=m_{r+i} p_{r+i}$ and $t=m p$ and by our definition of $\Gamma_{j}$ and $\epsilon,\left|p_{r+i}-k_{r+i} p\right|<\epsilon<p_{-} /(2 M)$ for some positive integer $k_{r+i}<M$, so we have that

$$
\begin{aligned}
\left|m_{r+i} k_{r+i}-m\right| p-m_{r+i}\left|k_{r+i} p-p_{r+i}\right| & \leq\left|m_{r+i}\left(p_{r+i}-k_{r+i} p\right)+m_{r+i} k_{r+i} p-m p\right| \\
& =\left|m p-m_{r+i} p_{r+i}\right|=\left|\tau_{r+i}-t\right|<\delta
\end{aligned}
$$

which shows that

$$
\left|m_{r+i} k_{r+i}-m\right| p<\delta+m_{r+i}\left|p_{r+i}-k_{r+i} p\right|<\delta+M \epsilon .
$$

But $\delta+M \epsilon<p$, since we have chosen $\delta<p_{-} / 2$ and $\epsilon<p_{-} /(2 M)$. This implies that $\left|m_{r+i} k_{r+i}-m\right|<1$ which is possible only if $m=m_{r+i} k_{r+i}$. This means that 
we must have, for $i=1, \ldots, v$, that $k_{r+i}\left[\hat{\gamma}_{j}\right]=q_{\alpha \beta_{*}}\left[\gamma_{i}^{\prime}\right]$. So we see that

$$
\begin{aligned}
c_{j}+\sum_{i=1}^{v} c_{r+i} k_{r+i} & =c \text { and consequently, } \\
\frac{c_{j}}{m}+\sum_{i=1}^{v} \frac{c_{r+i}}{m_{r+i}} & =\frac{c_{j}}{m}+\sum_{i=1}^{v} \frac{c_{r+i} k_{r+i}}{m_{r+i} k_{r+i}} \\
& =\frac{c_{j}}{m}+\frac{1}{m} \sum_{i=1}^{v} c_{r+i} k_{r+i} \\
& =\frac{1}{m}\left(c_{j}+\sum_{i=1}^{v} c_{r+i} k_{r+i}\right) \\
& =\frac{c}{m}
\end{aligned}
$$

which is what we wanted to prove. So we have independence from the covering, and so, finally, that the index is well-defined. We denote the index by $i(Y, \Phi, U)$.

Part 4: Verification of the properties of an index. Since the additivity and normalization property are trivially satisfied, it remains for us to establish homotopy invariance.

Let $U \subset Y \times[0, \infty) \times[0,1]$ be open and consider a continuous map

$$
\begin{aligned}
& \Xi: \operatorname{cl} U \rightarrow Y, \\
& (x, t, \lambda) \mapsto \Xi^{\lambda}(x, t)
\end{aligned}
$$

where for each $\lambda \in[0,1], \Xi^{\lambda}$ is a local semiflow with $\mathcal{D}\left(\Xi^{\lambda}\right) \subset \operatorname{cl} U^{\lambda}$ and such that $\Xi(x, t, \lambda) \neq x$ whenever $(x, t, \lambda) \in \partial U$. We have to show that $\lambda \mapsto i\left(Y, \Xi^{\lambda}, U^{\lambda}\right)$ is locally constant. Then let $\lambda \in[0,1]$. We choose a $\delta>0$ and a neighbourhood $V$ of $\{(x, t) \mid \Xi(x, t, \lambda)=x\}$ such that $\{(x, t) \mid \Xi(x, t, \mu)=x\} \subset V$ if $|\lambda-\mu|<\delta$. We then choose a convenient approximation $F_{\alpha}^{\lambda}$ for $\Xi^{\lambda}$ with distinguished orbits $\gamma_{1}, \ldots, \gamma_{r}$. By reducing $\delta$ if necessary we choose for $j \in\{1, \ldots, r\}$ a tube $T_{j} \times(\lambda-\delta, \lambda+\delta)$ around $\gamma_{j} \times\{\lambda\}$ for the local semiflow $\Xi^{\prime}$ given by $(x, t, \mu) \mapsto(\Xi(x, t, \mu), \mu)$ where $T_{j}$ is a tube around $\gamma_{j}$ for $\Xi^{\lambda}$ and such that $T_{j} \times\{\lambda\}$ may serve as $W_{j}$ in Condition 4 ) of the definition of a convenient approximation. If $\gamma_{j} \times\left\{t_{i}^{(j)}\right\} \subset P\left(\Xi^{\lambda}\right)$ for $j=1, \ldots, r$, $i=1, \ldots, k_{j}$, we let $\theta:=\frac{1}{4} \min \left\{\left|t_{i}^{(j)}-t_{l}^{(j)}\right| \mid 1 \leq i<l \leq k\right\}$. By further reducing $\delta$ if necessary, we may assume that for $|\lambda-\mu|<\delta$ and $(x, t) \in P\left(\Xi^{\mu}\right)$ with $x \in T_{j}$ we have that $\left|t-t_{i}^{(j)}\right|<\theta$ for some $i \in\left\{1, \ldots, k_{j}\right\}$. Then let $|\lambda-\mu|<\delta$. For definiteness, we assume that $\mu>\lambda$. If for some $j \in\{1, \ldots, r\}$ there is no periodic point of $\Xi^{\mu}$ in $T_{j} \times\{\mu\}$, this means that $I\left(N_{\alpha}, F_{\alpha}^{\lambda}, T_{j} \times(t-\delta, t+\delta)\right)=0$ since $\gamma_{j}^{\prime}$ can be removed by a small deformation.

So we choose an $s \leq r$ such that for $j \in\{1, \ldots, s\}$ there is a periodic orbit $\Gamma_{\mu}$ for $\Xi^{\mu}$ in $T_{j} \times\{\mu\}$ and for $j \in\{s+1, \ldots, r\}$ there is no such orbit. Then we extend $F_{\alpha}^{\lambda}$ over $Y \times[\lambda, \mu]$ : Let $\Sigma$ be a section of $\Xi^{\prime}$ at $x_{j} \in \gamma_{j} \times\{\lambda\}$. Choose $y \in \Sigma \cap\left(\Gamma_{j} \times\{\mu\}\right)$. If $\tau(y)=p(y)$, we may assume that $p_{\alpha}$ maps $\Gamma_{j}$ homeomorphically onto $\gamma_{j}^{\prime}$. If $p(y)=\tau_{1}(y)+\cdots+\tau_{m}(y)$ for some $m>1$, we have that $p_{\alpha} \mid \Gamma_{j}: \Gamma_{j} \rightarrow \gamma_{j}^{\prime}$ is an $m$ fold covering. In this case we say that the minimal period of $\Gamma_{j}$ is roughly $m$ times the minimal period of $\gamma_{j}$. For $\nu \in[\lambda, \mu]$ we define $q_{\alpha}((x, \nu)):=\left(p_{\alpha} x, \nu\right)$. For $y \in N_{\alpha}$ we let $i_{\alpha}^{\prime}$ map $\gamma_{j}^{\prime} \times\{\mu\}$ homeomorphically onto $\Gamma_{j} \times\{\mu\}$ such that $q_{\alpha} \circ i_{\alpha}^{\prime}=\mathrm{id}$, and then we extend $i_{\alpha}^{\prime}$ as a full realization over $N_{\alpha} \times[\lambda, \mu]$. (Strictly speaking, 
$N_{\alpha} \times[\lambda, \mu]$ is not a simplicial complex, but it is obviously triangulable.) We may then extend $F_{\alpha}$ over $N_{\alpha} \times[\lambda, \mu]$ in such a way that $F_{\alpha} \mid N_{\alpha} \times\{\mu\}$ is a convenient approximation to $\Xi^{\mu}$ (with the exception that Condition 2) in the definition of a convenient approximation has to be modified as indicated above). Moreover, the construction of a convenient approximation will show that for $\nu \in(\lambda, \mu)$ the projection onto $N_{\alpha}$ of the set $P_{\alpha}^{\nu}:=\left\{(x, t) \mid F_{\alpha}(x, t, \nu)=(x, \nu)\right\}$ is contained in $T_{j}$ or in some contractible set. By the homotopy invariance for the topological index $I(Y, \Phi, U)$ we have that

$$
I\left(N_{\alpha}, F_{\alpha}^{\lambda}, T_{j} \times\left(t_{i}^{(j)}-\delta, t_{i}^{(j)}+\delta\right)\right)=I\left(N_{\alpha}, F_{\alpha}^{\mu}, T_{j} \times\left(t_{i}^{(j)}-\delta, t_{i}^{(j)}+\delta\right)\right) .
$$

Let $j \in\{1, \ldots, r\}, i \in\left\{1, \ldots, k_{i}\right\}$, let $t:=t_{i}^{(j)}$, and assume that $\Gamma_{j} \times\left\{t^{\prime}\right\} \subset P\left(\Xi^{\mu}\right)$ for some $\left|t-t^{\prime}\right|<\theta$. If there is no such $t^{\prime}$, then both sides of the last equation are zero. If $I\left(N_{\alpha}, F_{\alpha}^{\lambda}, T_{j} \times(t-\delta, t+\delta)\right)=c_{t}\left[\gamma_{j}\right]$ and $m\left(\gamma_{j}, t\right)=: m_{t}$, then $\gamma_{j} \times\{t\}$ contributes $c_{t} / m_{t}$ to $i\left(Y, \Xi^{\lambda}, U\right)$ and we have that $I\left(N_{\alpha}, F_{\alpha}^{\mu}, T_{j} \times\left(t^{\prime}-\delta, t^{\prime}+\delta\right)\right)=$ $c_{t}\left[\gamma_{j}\right]$. If the minimal period of $\Gamma_{j}$ is roughly $m$ times the minimal period of $\gamma_{j}$, we have that $\left[\Gamma_{j}\right]=m\left[\gamma_{j}\right]$ and $m \cdot m\left(\Gamma_{j}, t^{\prime}\right)=m_{t}$. So we have that

$$
I\left(N_{\alpha}, F_{\alpha}^{\mu}, T_{j} \times\left(t^{\prime}-\delta, t^{\prime}+\delta\right)\right)=\frac{c_{t}}{m}\left[\Gamma_{j}\right]
$$

and $\Gamma_{j}$ contributes $c_{t} /\left[m \cdot m\left(\Gamma_{j}, t^{\prime}\right)\right]$ to $i\left(Y, \Xi^{\mu}, U\right)$. This shows that the index is locally constant. So our index is well-defined and enjoys the required properties.

\section{Appendix: Existence of a CONVEnient approximation}

It still remains for us to establish the existence of arbitrarily fine convenient approximations and to indicate the necessary modifications in order to justify the arguments we used in proving independence from the covering and homotopy invariance.

Proposition. Let $Y$ be a compact connected ANR, $U$ open in $Y \times[0, \infty)$ and $\Phi$ a local semiflow on $Y$ such that $\operatorname{cl} U \subset \mathcal{D}(\Phi)$ and $P:=P(\Phi, U) \subset U$. Let $\beta^{\prime}$ be an open cover of $Y$. Then there exist a finite open refinement $\alpha$ of $\beta^{\prime}$ and a convenient approximation $f_{\alpha}: j_{\alpha}^{-1}(U) \rightarrow N_{\alpha}$.

Proof. We choose a finite open refinement $\kappa$ of $\beta^{\prime}$ such that $I\left(N_{\alpha}, p_{\alpha} \Phi j_{\alpha}, j_{\alpha}^{-1}(U)\right)$ is defined and independent from the choices involved if $\alpha$ refines $\kappa$. More precisely: In [F2] it is shown that we may choose $\kappa$ so fine that $I\left(N_{\alpha}, p_{\alpha} \Phi j_{\alpha}, j_{\alpha}^{-1}(U)\right)$ is defined and depends neither on $\alpha$ nor on $p_{\alpha}$ or $i_{\alpha}$ provided $i_{\alpha}$ has mesh $\kappa$. Choose a finite open cover $\beta$ such that each partial realization $i: K \rightarrow Y$ of a finite simplicial complex $K$ which has mesh $\beta$ extends to a full realization $I: K \rightarrow Y$ which has mesh $\kappa$. (This is possible since $Y$ is an ANR - cf. [H], IV, Theorem 4.1.) Choose a finite open star refinement $\beta^{*}$ of $\beta$. Now let $\gamma$ be a periodic orbit of $\Phi$ in $\operatorname{pr}_{1} P$ with tube $T_{\gamma}$ and section $S(\gamma)$ at $x \in \gamma$. We then choose a positive integer $N$ such that for $i=0, \ldots, N-1$ we have that $\left\{\phi_{s} x \mid \frac{i}{N} p(x) \leq s \leq \frac{i+1}{N} p(x)\right\}$ is contained in an element of $\beta^{*}$. Then we choose a neighbourhood $W(\gamma)$ of $\gamma$ such that for each periodic point $x \in W(\gamma)$ there is a positive integer $k \leq M$ such that $|p(x)-k p(\gamma)|<p_{-} /(2 M)$. This will ensure that the first part of Condition 2) in the definition of a convenient approximation holds true. Moreover, we choose $W(\gamma)$ so small that there are a retraction $r_{\gamma}: W(\gamma) \rightarrow \gamma$ and an $\epsilon>0$ such that the following holds: Let $T_{0}^{\prime}:=T_{N}^{\prime}:=\Phi\left(\{x\} \times\left((p(x)-\epsilon, 0] \cup\left[0, \frac{p(x)}{N}+\epsilon\right)\right)\right)$ and $T_{i}^{\prime}:=\Phi\left(\{x\} \times\left(\frac{i}{N} p(x)-\epsilon, \frac{i+1}{N} p(x)+\epsilon\right)\right)$ for $i=1, \ldots, N-1$. Then, for 
$i=0, \ldots, N$, each of the sets $T_{i}(\gamma):=r^{-1}\left(T_{i}^{\prime}\right)$ is contained in an element of $\beta^{*}$ and $T_{i}(\gamma) \cap T_{j}(\gamma) \neq \emptyset$ only if $|i-j| \leq 1$. Call $T(\gamma):=T(x):=\bigcup_{i=0}^{N-1} T_{i}(\gamma)$.

We now select $\gamma_{1}, \ldots, \gamma_{s}$ in $\operatorname{pr}_{1} P$ and points $x_{i} \in \gamma_{i}$ such that $T\left(x_{1}\right), \ldots, T\left(x_{s}\right)$ cover $\operatorname{pr}_{1} P$. In order to simplify our notation we may assume that we may use the same $N$ for $T\left(x_{1}\right), \ldots, T\left(x_{s}\right)$. By reducing the size of the $T\left(x_{i}\right)$ we may further assume that $\gamma_{i} \cap T\left(x_{j}\right)=\emptyset$ whenever $i \neq j$. Then there are neighbourhoods $U_{i}^{\prime} \subset \operatorname{cl} U_{i}^{\prime} \subset U_{i} \subset \operatorname{cl} U_{i} \subset T\left(x_{i}\right)$ of $\gamma_{i}$ such that the $U_{i}^{\prime}$ still cover $\operatorname{pr}_{1} P$. We then select disjoint neighbourhoods $V_{i}^{\prime} \subset \operatorname{cl} V_{i}^{\prime} \subset V_{i} \subset \operatorname{cl} V_{i} \subset U_{i}^{\prime}$ of $\gamma_{i}$ such that $\Phi\left(\operatorname{cl} V_{i}^{\prime} \times\left[0, t_{+}\right]\right) \subset V_{i}$ and call $r_{j}:=r_{\gamma_{j}} \mid V_{j}$.

The set $\operatorname{pr}_{1} P$ is then covered by $\alpha_{1}:=\left\{T_{i}\left(x_{j}\right) \mid j=1, \ldots, s, i=0, \ldots, N-1\right\}$. We now select a finite open cover $\alpha_{2}$ of $Y \backslash \bigcup_{j=1}^{s} U_{j}$ consisting of open subsets of $Y \backslash \bigcup_{j=1}^{s} \operatorname{cl} U_{j}^{\prime}$ such that the following holds:

1. If $V \in \alpha_{2}$, then there is a $W \in \beta^{*}$ such that $W \cap \operatorname{pr}_{1} P=\emptyset$ and $\operatorname{st}_{\alpha_{2}} V \subset W$.

2. If $V \in \alpha_{2}$ and $U \cap \operatorname{pr}_{1}^{-1} V \neq \emptyset$, then $V \cap \operatorname{pr}_{1} P=\emptyset$.

3. If $V \in \alpha_{2}$ and $U \cap \operatorname{pr}_{1}^{-1} V \neq \emptyset$, then $\Phi\left(\right.$ st $\left.V \times\left[t_{-}, t_{+}\right]\right) \cap \mathrm{st}_{\alpha_{2}} V=\emptyset$.

Let $\alpha_{0}$ be the cover of $Y$ which consists of $\alpha_{1}$ and $\alpha_{2}$. For each maximal simplex $\sigma=\left\langle U_{1}, \ldots, U_{n}\right\rangle$ of $A_{0}$ we choose an open set $U_{\sigma}^{\prime} \subset \operatorname{cl} U_{\sigma}^{\prime} \subset \bigcap_{i=1}^{n} U_{i}$ such that $\operatorname{cl} U_{\sigma}^{\prime}$ does not meet $\bigcup_{j=1}^{s} V_{j}$. If $\sigma_{0}$ is a maximal simplex of $A_{0}$ and $\sigma_{1}, \ldots, \sigma_{m}$ are the maximal simplices in $A_{0}$ which meet $\sigma_{0}$, we let $U_{\sigma_{0}}:=\bigcup_{i=0}^{m} U_{\sigma_{i}}^{\prime}$. Call $\alpha^{*}$ the set of all $U_{\sigma}$ corresponding to maximal simplices $\sigma$ of $A_{0}$, let $\alpha:=\alpha_{0} \cup \alpha^{*}$, and denote by $A_{*}$ the subcomplex of $N_{\alpha}$ containing all $\left\langle U_{\sigma}\right\rangle$. $\alpha$ will then be a refinement of $\beta$. With this choice of covering we can satisfy Conditions 1) a) - c): If $\sigma_{1}, \ldots, \sigma_{m}$ are maximal simplices in $A_{0}$ each of which meets the maximal simplex $\sigma_{0}$ of $A_{0}$, then each $U_{\sigma_{i}}$ contains $U_{\sigma_{0}}^{\prime}$; hence $\bigcap_{i=0}^{m} U_{\sigma_{i}} \neq \emptyset$. Conversely, assume that $\bigcap_{i=1}^{m} U_{\sigma_{i}} \neq \emptyset$. Now each $U_{\sigma_{i}}$ is a disjoint union of sets $U_{\sigma_{j}}^{\prime}$, so there must be a maximal simplex $\sigma$ in $A_{0}$ such that $U_{\sigma}^{\prime} \subset U_{\sigma_{i}}$ for $i=1, \ldots, m$. By our construction, this means that $\sigma$ meets $\sigma_{i}$. This establishes 1) a). As to 1 ) b) we retain the notation of that condition. The assumption is that $\emptyset \neq \bigcap_{\langle V\rangle \in E^{\prime}} V$. But each $U_{\sigma_{i}}$ is made up of pieces (viz., the $U_{\sigma_{j}}^{\prime}$ ) which are contained in a maximal intersection. So this can happen only if $\bigcap_{i=1}^{m} U_{\sigma_{i}}$ meets the (maximal) intersection $\bigcap_{\langle V\rangle \in E} V$ and $\bigcap_{\langle V\rangle \in E^{\prime}} V=\bigcap_{\langle V\rangle \in E} V$. Finally, we have seen already that we can fulfill 1) c) whenever we can fulfill 1) a) and $b$ ).

We will now construct a canonical projection $p_{\alpha}: Y \rightarrow N_{\alpha}$ which maps $\gamma_{1}, \ldots, \gamma_{s}$ homeomorphically into the one-skeleton of $A_{0}$. Then let $y \in V_{j}$. As before, we fix the standard euclidean metric $d$ on $N_{\alpha}$. If $r_{j} y=\phi_{t} x_{j}$ with $0 \leq t \leq p\left(x_{j}\right)$, write $t=(\lambda+i) p\left(x_{j}\right) / N$ with $0 \leq \lambda \leq 1$ and $0 \leq i \leq N-1$ and let $p_{\alpha}^{\prime}(y):=$ $(1-\lambda)\left\langle T_{i}\left(x_{j}\right)\right\rangle_{\alpha}+\lambda\left\langle T_{i+1}\left(x_{j}\right)\right\rangle_{\alpha}$. This defines a canonical projection $p_{\alpha}^{\prime}: \bigcup_{j=1}^{s} V_{j} \rightarrow$ $N_{\alpha}$. Denote by $p_{\alpha}^{\prime \prime}: Y \rightarrow N_{\alpha}$ any canonical projection constructed as in [Du], (cf. supra, loc. cit.) and choose a continuous function $\lambda: Y \rightarrow[0,1]$ such $\lambda \mid \bigcup_{j=1}^{s} V_{j}^{\prime}=0$ and $\lambda \mid \bigcap_{j=1}^{s}\left(Y \backslash V_{j}\right)=1$. If $x \in V_{j}$, we have that $p_{\alpha}^{\prime} x$ and $p_{\alpha}^{\prime \prime} x$ belong to a common simplex, so we define $p_{\alpha}(x):=(1-\lambda(x)) p_{\alpha}^{\prime}(x)+\lambda(x) p_{\alpha}^{\prime \prime}(x)$. On $\bigcap_{j=1}^{s}\left(Y \backslash V_{j}\right)$ we let, of course, $p_{\alpha}:=p_{\alpha}^{\prime \prime}$. Obviously, $p_{\alpha}$ maps each $\gamma_{j}$ homeomorphically onto $\gamma_{j}^{\prime}:=p_{\alpha}\left(\gamma_{j}\right)$ (observe that $p_{\alpha} \mid \gamma_{j}=p_{\alpha}^{\prime}$ ). On the other hand, we now define $i_{\alpha}: N_{\alpha} \rightarrow Y$ in the following way: First, for $i=1, \ldots, N-1, j=1, \ldots, s$, and $0 \leq \lambda \leq 1$ we map $(1-\lambda)\left\langle T_{i}\left(x_{j}\right)\right\rangle_{\alpha}+\lambda\left\langle T_{i+1}\left(x_{j}\right)\right\rangle_{\alpha}$ to $\Phi\left(x_{j}, \frac{i+\lambda}{N} p\left(x_{j}\right)\right)$. For other vertices $\langle U\rangle_{\alpha} \in A_{0}$ we map $\langle U\rangle_{\alpha}$ to an arbitrary point of $U$. If $\sigma$ is a maximal simplex of $N_{\alpha}$, we map $\left\langle U_{\sigma}\right\rangle_{\alpha}$ to a point of $U_{\sigma}$. So we have a partial map 
$i_{\alpha}: N_{\alpha} \rightarrow Y$ which has mesh $\beta$ and we extend this partial map to a full realization $i_{\alpha}: N_{\alpha} \rightarrow Y$ of mesh $\kappa$. So all requirements of Condition 2) are fulfilled. As we have explained in the discussion after the definition of a convenient covering this choice of covering and canonical projection will also satisfy Condition 1).

For $(x, t) \in j_{\alpha}^{-1}(\mathrm{cl} U)$ we define $\psi_{t} x:=p_{\alpha} \phi_{t} i_{\alpha} x$. Choose neighbourhoods $D \subset$ cl $D \subset B$ of $A_{*}$ such that $B \cap\left\{\psi_{t} x \mid(x, t) \in j_{\alpha}^{-1}(\operatorname{cl} U)\right\}=\emptyset$ which is possible since $A_{*}$ does not meet the range of $p_{\alpha}$. Now let

$$
\nu_{1}:=\inf \left\{d\left(x, \psi_{t} x\right) \mid\left(i_{\alpha} x, t\right) \in \operatorname{cl} U \quad \text { and } \quad i_{\alpha}(x) \in Y \backslash \bigcup_{j=1}^{s} U_{j}\right\}
$$

and

$$
\nu:=\min \left\{\nu_{1}, d\left(A_{*}, N_{\alpha} \backslash D\right), \inf \left\{d\left(x, \psi_{t} x\right) \mid\left(i_{\alpha} x, t\right) \in \partial U\right\}\right\}
$$

For $x \in N_{\alpha}$ we denote by $\epsilon(x)$ the maximal distance $d(x, v)$ where $v$ is a vertex of a simplex containing $x$. Obviously, $\epsilon$ is continuous. Then let $d(x, y)<\epsilon(x)$. Then either $y$ belongs to the same simplex as $x$ or $y$ must belong to a simplex sharing a face with a simplex containing $x$, since $\epsilon(x)<\sqrt{2}$ and the diameter of a simplex having more than one point is $\sqrt{2}$. We then let $\delta_{0}:=\min \left\{\nu, \epsilon(x) \mid x \in N_{\alpha} \backslash D\right\}$.

The next lemma describes a uniform way to move points in $N_{\alpha} \backslash D$ in the direction of the "redundant" vertices:

Lemma. There is a continuous function $\zeta: N_{\alpha} \backslash D \times\left[\frac{\delta_{0}}{16}, \frac{3 \delta_{0}}{8}\right] \rightarrow N_{\alpha}$ such that (with $\zeta_{s}(x):=\zeta(x, s)$ ) we have that, for $x \in N_{\alpha} \backslash D$ and $s \in\left[\frac{\delta_{0}}{16}, \frac{3 \delta_{0}}{8}\right]$,

a) $d\left(x, \zeta_{s}(x)\right)=s$.

b) $d\left(\zeta_{s}(x), A_{*}\right)<d\left(x, A_{*}\right)$.

c) If $x \in A_{0}$ belongs to a face of a simplex $\sigma$ in $N_{\alpha}$ which is not maximal, then $\zeta_{s}(x) \notin \sigma$ for $s \in\left(\frac{\delta_{0}}{16}, \frac{3 \delta_{0}}{8}\right]$, but $\zeta_{s}(x)$ belongs to a maximal simplex containing $x$.

Proof. In a first step, we construct an auxiliary map $\eta: A_{0} \rightarrow N_{\alpha}$ which we will later use as $\zeta_{\delta_{0} / 16}$ on $A_{0}$. We proceed by induction. Let $\langle V\rangle_{\alpha} \in A_{0}$. If $\langle V\rangle_{\alpha}$ is a common vertex of the maximal simplices $\sigma_{1}, \ldots, \sigma_{m}$ of $A_{0}(m=1$ possible), we denote by $b$ the barycenter of $\left\langle U_{\sigma_{1}}, \ldots, U_{\sigma_{m}}\right\rangle_{\alpha}$ and choose $\eta\left(\langle V\rangle_{\alpha}\right)$ as the point on $\left[\langle V\rangle_{\alpha}, b\right]$ which is at distance $\delta_{0} / 16$ from $\langle V\rangle_{\alpha}$. Since we have fulfilled Condition 1) b) in Definition 2, $\eta(x)$ will then be a point on a proper face. Suppose then that $\eta$ is defined on the boundary of a simplex $\sigma$ in $A_{0}$ which is a common face of the maximal simplices $\Sigma_{1}, \ldots, \Sigma_{m}$ of $A_{0}$ such that, for $x$ on the boundary of $\sigma, \eta(x)$ is a point on a proper face of a simplex containing $\sigma$ and $\left\langle U_{\Sigma_{1}}, \ldots, U_{\Sigma_{m}}\right\rangle_{\alpha}$ with $d(x, \eta(x))=\delta_{0} / 16$ and $d\left(\eta(x), A_{*}\right)<d\left(x, A_{*}\right)$. Call $b$ the barycenter of $\sigma$ and $B$ the barycenter of $\left\langle U_{\Sigma_{1}}, \ldots, U_{\Sigma_{m}}\right\rangle_{\alpha}$. Then let $x$ be an interior point of $\sigma$. If $x=b$, define $\eta(b)$ as the unique point on the straight line segment $[b, B]$ which is at distance $\delta_{0} / 16$ from $b$. If $x \neq b$, write $x=(1-\lambda) y+\lambda b$ with $0<\lambda<1$ and $y$ on a face of $\sigma$. We then let $\xi=(1-\lambda) \eta(y)+\lambda \eta(b)$ which makes sense by the induction hypothesis. We then define $\eta(x)$ as the point $x+\mu(\xi-x)$ which is at distance $\delta_{0} / 16$ from $x$. Again by Condition 1) b) in Definition 2, $\eta(x)$ is a point on a proper face if $\sigma$ is not a maximal simplex of $A_{0}$. We now define $\zeta_{s}(x)$ if $x \in N_{\alpha} \backslash D \backslash A_{0}$ and $0<s \leq 3 \delta_{0} / 8$. If $x$ belongs to a simplex $\tau$ which is not maximal we will define $\zeta_{s}(x)$ in such a way that $\zeta_{s}(x)$ is an interior point of a maximal simplex of $N_{\alpha}$. Choose a maximal simplex $\sigma$ which has $\tau$ as a face. Choose a point $z \in \tau$ which is not on a face of $\tau$ such that $z \in \partial D$ and choose an interior point $z^{\prime} \in \sigma$ such 
that $d\left(z^{\prime}, A_{*}\right)<d(z, A)$ and $d\left(z, z^{\prime}\right)=3 \delta_{0} / 8$. Now there are a point $y \in \tau \cap A_{0}$ and a $\lambda \in(0,1)$ such that $x=(1-\lambda) y+\lambda z$. Let $x^{\prime}=(1-\lambda) \eta(y)+\lambda z^{\prime}$. We then define $\zeta_{s}(x)$ as the point $x+\mu\left(x^{\prime}-x\right)$ which is at distance $s$ from $x$. We now define $\zeta_{s}(x)$ for $x \in A_{0}$ and $\delta_{0} / 16 \leq s \leq 3 \delta_{0} / 8$. If $s=\delta_{0} / 16$, we let $\zeta_{s}(x)=\eta(x)$. If $\delta_{0} / 16<s \leq 3 \delta_{0} / 8$, we choose $t>0$ in such a way that $\zeta_{s}(x):=\zeta_{t}(\eta(x))$ is at distance $s$ from $x$. Finally, we extend $\zeta_{s}$ over the interior points of maximal simplices: if $\sigma$ is a maximal simplex with barycenter $b$, we define $\zeta_{s}(b)$ to be the point on $\left[b,\left\langle U_{\sigma}\right\rangle\right]$ which is at distance $s$ from $b$. If $x \neq b$ is an interior point of $\sigma$, we write $x=(1-\lambda) y+\lambda b$ with $0<\lambda<1$ and $y$ on the boundary of $\sigma$. We then let $\xi=\ell\left(\zeta_{s}(y), \zeta_{s}(b) ; \lambda\right)$ where $\ell$ is defined as in 3.1. Call $\tau$ the maximal simplex containing $\zeta_{s}(y)$ (so either $\tau=\sigma$ or $\tau$ is adjacent to $\sigma$ ). Then we define $\zeta_{s}(x)$ to be the point on $\left[\xi,(1-\lambda)\left\langle U_{\tau}\right\rangle_{\alpha}+\lambda\left\langle U_{\sigma}\right\rangle_{\alpha}\right]$ which is at distance $s$ from $x$.

Remark. For later purposes we sketch a modification of the construction of $\zeta$ in the situation of section 3.4 in the proof of the theorem. So we assume that $\alpha$ and $\beta$ are as in 3.4. If we can find functions $\zeta^{0}$ for $N_{\alpha}$ and $\zeta^{1}$ for $N_{\beta}$ as in the lemma such that for $(x, s) \in N_{\alpha} \times\left[\frac{\delta_{0}}{16}, \frac{3 \delta_{0}}{8}\right]$ we have that $\zeta_{s}^{0}(x)$ and $\zeta_{s}^{1}\left(q_{\alpha \beta} x\right)$ are contained in a common simplex of $N_{\alpha \cup \beta}$, we may define $\zeta_{s}(x, t):=(1-t) \zeta_{s}^{0}(x)+t \zeta_{s}^{1}\left(q_{\alpha \beta}(x)\right)$. So we start by fixing functions $\eta^{0}$ for $A_{0}$ and $\eta^{1}$ for $B_{0}:=\left\{\langle W\rangle \mid W \in \beta_{0}\right\}$ as in the proof of the lemma. If $x \in A_{0}$ and if $\eta^{0}(x)$ belongs to a simplex $\tau$, then $\eta^{1}\left(q_{\alpha \beta} x\right)$ belongs to a simplex containing $q_{\alpha \beta}(\tau)$. There is only one point in the definition of $\zeta$ where we made an arbitrary choice, namely if $x \in N_{\alpha} \backslash A_{0} \backslash D$ belongs to a simplex $\tau$ which is not maximal. There are then two cases: either there is a maximal simplex $\sigma \supset \tau$ such that $q_{\alpha \beta}(\tau)$ is a proper face of $q_{\alpha \beta}(\sigma)$. Then we choose $\zeta_{s}^{0}(x)$ to be an interior point of $\sigma$ and $\zeta_{s}^{1}\left(q_{\alpha \beta} x\right)$ as an interior point of a maximal simplex $\sigma^{\prime} \supset q_{\alpha \beta}(\sigma)$. Or, for all maximal simplices $\sigma \supset \tau$ we have that $q_{\alpha \beta}(\sigma)=q_{\alpha \beta}(\tau)$. In this case, we choose a maximal simplex $\sigma \supset \tau$ and a maximal simplex $\sigma^{\prime} \supset q_{\alpha \beta}(\tau)$ and then $\zeta_{s}^{0}(x)$ and $\zeta_{s}^{1}\left(q_{\alpha \beta} x\right)$ as interior points of $\sigma$ and $\sigma^{\prime}$, respectively. In both cases, $\zeta_{s}^{0}(x)$ and $\zeta_{s}^{1}\left(q_{\alpha \beta} x\right)$ will lie in a common simplex of $N_{\alpha \cup \beta}$. After we have exercised this choice $\zeta^{0}$ and $\zeta^{1}$ are completely determined by the above proof.

We now fix a function $\zeta$ for $N_{\alpha}$ as in the lemma. With the aid of $\zeta$ we will now define a convenient approximation for $\psi$ on $j_{\alpha}^{-1}(\operatorname{cl} U)$. Then let $(x, t) \in j_{\alpha}^{-1}(\operatorname{cl} U)$ and define $\rho(x, t):=d\left(x, \psi_{t} x\right)$.

1. First, if $\rho(x, t)$ is large enough, we simply use the given $\psi_{t}$ : If $\rho(x, t) \geq \delta_{0} / 8$, we let $f_{\alpha}(x, t):=\psi_{t} x$. We then have that $f_{\alpha}(x, t)=\psi_{t} x$ if $x \in D$ (by our choice of $\nu$ and hence of $\left.\delta_{0}\right)$ or if $x \notin \bigcup_{j=1}^{s} W_{j}$ or if $(x, t) \in \partial j_{\alpha}^{-1}(U)$.

2. If $\rho(x, t)$ is very small, we use $\zeta$ to move $\psi_{t} x$ towards $A_{*}$ in such a way that we avoid the point $x$ and that periodic points on the distinguished orbits are not moved at all: We choose a continuous function $\lambda: N_{\alpha} \rightarrow\left[0, \delta_{0} / 4\right]$ such that $\lambda(x)=0$ iff $x \in \bigcup_{j=1}^{s} \gamma_{j}^{\prime}$. Then we choose a continuous function $\Lambda: j_{\alpha}^{-1}(\operatorname{cl} U) \rightarrow[0,1]$ such that $\Lambda(x, t)=0$ iff $\rho(x, t) \geq \delta_{0} / 8$ and $\Lambda(x, t)=1$ iff $\rho(x, t) \leq \delta_{0} / 16$. If $\rho(x, t) \leq \delta_{0} / 16$, we let $f_{\alpha}(x, t)=\zeta_{\rho(x, t)+\lambda(x)}\left(\psi_{t} x\right)$. If $x \in A_{0}$, we can have $f_{\alpha}(x, t)=x$ only if $\rho(x, t)=0$ (hence $\psi_{t} x=x$ ) and $\lambda(x)=0$, so $x \in \bigcup_{j=1}^{s} \gamma_{j}^{\prime}$. But then $\psi_{t} x=x$ only if $t$ is a period of $x$. If $x \notin A_{0}$, we have that $d\left(x, f_{\alpha}(x, t)\right) \geq \rho(x, t)+$ $\lambda(x)-\rho(x, t)=\lambda(x)>0$. Moreover, $x$ is on the sphere of radius $\rho(x, t)$ around $\psi_{t} x$, so $d\left(\zeta_{\rho(x, t)+\lambda(x)}\left(\psi_{t} x\right), A_{*}\right)<d\left(x, A_{*}\right)$. Since $f_{\alpha}(x, t)=x$ for $x \in \bigcup_{j=1}^{s} \gamma_{j}^{\prime}$ and $t$ a period of $x$, there are a neighbourhood $W_{j}$ of $x$ and a $\delta>0$ such that $\rho(x, t)<\delta_{0} / 16$ if $x \in W_{j}$ and $\left|t-t^{\prime}\right|<\delta$ for a period $t$ of $x$. This shows that we 
can fulfill Condition 4). Condition 5) holds by our choice of $\alpha$ and the definition of $I\left(Y, \Phi, j_{\alpha}^{-1}(V)\right)$.

Finally, we have to deal with the difficult situation where $\delta_{0} / 16 \leq \rho(x, t) \leq \delta_{0} / 8$. If $x \in A_{0}$, we simply move the point $\psi_{t} x$ to $\zeta_{\rho(x, t)+\lambda(x)}\left(\psi_{t} x\right)$ on the line joining these points:

3. If $x \in A_{0}$ and $\rho(x, t) \leq \delta_{0} / 8$, we have that $\psi_{t} x$ and $\zeta_{\rho(x, t)+\lambda(x)}\left(\psi_{t} x\right)$ belong to the same simplex, so we may define $f_{\alpha}(x, t):=(1-\Lambda(x, t)) \psi_{t} x+$ $\Lambda(x, t) \zeta_{\rho(x, t)+\lambda(x)}\left(\psi_{t} x\right)$. For $\rho(x, t)=\delta_{0} / 8$ we have that $\Lambda(x, t)=0$, hence $f_{\alpha}(x, t)$ $=\psi_{t} x$, and for $\rho(x, t) \leq \delta_{0} / 16$ we have that $\Lambda(x, t)=1$, so the definition agrees with the one given above. As before, we can have $f_{\alpha}(x, t)=x$ only if $\Lambda(x, t)=0$ (but then $f_{\alpha}(x, t)=\psi_{t} x$ and $\left.\rho(x, t) \geq \delta_{0} / 8\right)$ or if $\rho(x, t)+\lambda(x)=0$ but this is the situation we discussed in 2).

At this stage of the proof we observe that for $j=1, \ldots, s$ and $t \in M_{j}$ we have that $\gamma_{j}^{\prime} \times\{t\}$ is isolated in $P_{\alpha}$ provided we define $f_{\alpha}$ in a continuous way: If $x \in A_{0}$, we have that $f_{\alpha}(x, t)=x$ only if $x$ is on some $\gamma_{j}^{\prime}$ and $t \in M_{j}$. So there are a neighbourhood $\Omega_{j}$ of $\gamma_{j}^{\prime}$ and a $\delta_{j}>0$ such that $\rho(x, s)<\delta_{0} / 16$ if $x \in \operatorname{cl} \Omega_{j}$ and $|t-s| \leq \delta_{j}$ for some $t \in M_{j}$. If $x \in A_{0} \cap \operatorname{cl} \Omega_{j}$ and $|t-s| \geq \delta_{j}$ for all $t \in M_{j}$, we have that $f_{\alpha}(x, s) \neq x$ by our construction in 1), 2), and 3). So there is a neighbourhood $\Omega_{j}^{\prime} \subset \Omega_{j}$ of $\gamma_{j}^{\prime}$ such that $f_{\alpha}(x, s) \neq x$ whenever $x \in \operatorname{cl} \Omega_{j}^{\prime}$ and $|t-s| \geq \delta_{j}$ for all $t \in M_{j}$. Now $f_{\alpha}(x, s) \neq x$ whenever $x \in A_{0} \backslash \bigcup_{j=1}^{s} \gamma_{j}^{\prime}$ and $(x, s) \in \operatorname{cl} j_{\alpha}^{-1}(U)$, so there is a neighbourhood $V^{\prime}$ of $A_{0} \backslash \bigcup_{j=1}^{s} \mathrm{cl} \Omega_{j}^{\prime}$ such that $f_{\alpha}(x, s) \neq x$ whenever $x \in V^{\prime}$ and $(x, s) \in \operatorname{cl} j_{\alpha}^{-1}(U)$. But then there is a neighbourhood $V$ of $A_{0}$ such that $f_{\alpha}(x, t)=x$ for $x \in V$ only if $x$ is on some $\gamma_{j}^{\prime}$ and $t \in M_{j}$. This shows that the $\gamma_{j}^{\prime} \times\{t\}$ are isolated components of $P_{\alpha}$. We will now define $f_{\alpha}$ in such a way that $f_{\alpha}(x, t) \neq x$ if $x \notin A_{0}$ and if the intersection of $A_{0}$ and the minimal simplex $\sigma$ containing $x$ is either empty or a proper face in $A_{0}$. If we succeed in doing so, Condition 3) will be satisfied since we will have that $f_{\alpha}(x, t)=x$ for $x \notin A_{0}$ can happen only if the minimal simplex $\sigma$ containing $x$ has vertices both in $A_{0}$ and in $A_{*}$ and $\sigma \cap A_{0}$ is maximal in $A_{0}$. But this obviously implies that each component of these $x$ is contained in a contractible set. So let $x \notin A_{0}$ be such that the minimal simplex containing $x$ meets $A_{0}$ in a proper face. We then draw a small sphere around $x$ and join $\psi_{t} x$ to the nearest point on this sphere; then we move on a great circle to the point which is nearest to $\zeta_{\rho(x, t)+\lambda(x)}\left(\psi_{t} x\right)$, and then again linearly to this last point.

4. Let $\delta_{0} / 16 \leq \rho(x, t) \leq \delta_{0} / 8, x \notin A_{0}$ and assume that the intersection with $A_{0}$ of the minimal simplex $\sigma$ containing $x$ is either empty or a proper face. As before, we have that $x \neq \zeta_{\rho(x, t)+\lambda(x)}\left(\psi_{t} x\right)$. Call $\mu(x)$ the point in $A_{0}$ which is nearest to $x$ $($ so $\mu(x) \in \sigma)$. We let

$$
r(x, t):=\min \left\{d\left(x, A_{0}\right), \rho(x, t), d\left(\psi_{t} x, \mu(x)\right), d\left(x, \zeta_{\rho(x, t)+\lambda(x)}\left(\psi_{t} x\right)\right)\right\} .
$$

So, under the present assumptions, we have that $r(x, t)=0$ iff $\psi_{t} x=\mu(x)$. Note that Condition c) in the lemma implies $\zeta_{\rho(x, t)+\lambda(x)}(\mu(x)) \notin \sigma$ if $\rho(x, t)+\lambda(x)>$ $\delta_{0} / 16$ since we have already seen that $\sigma$ cannot be maximal. Since we are dealing with the case $\rho(x, t) \geq \delta_{0} / 16$ we can have $\rho(x, t)+\lambda(x)=\delta_{0} / 16$ only if $\rho(x, t)=$ $\delta_{0} / 16$ and $\lambda(x)=0$. But then $f_{\alpha}(x, t)$ has already been defined in 2 . Define $\ell$ as in 3.1 and let

$$
\theta(x, t):=\min \left\{d\left(x, \ell\left(\psi_{t} x, \zeta_{\rho(x, t)+\lambda(x)}\left(\psi_{t} x\right) ; \lambda\right)\right) \mid 0 \leq \lambda \leq 1\right\}
$$


So $r(x, t)=0$ implies $\theta(x, t)>0$. If $r(x, t) \leq \theta(x, t)$, we let

$$
f_{\alpha}(x, t):=\ell\left(\psi_{t} x, \zeta_{\rho(x, t)+\lambda(x)}\left(\psi_{t} x\right) ; \Lambda(x, t)\right) .
$$

If $0 \leq \theta(x, t) \leq r(x, t) / 2$, we call $\psi_{t}^{\prime} x$ the point $\ell\left(\psi_{t} x, x ; \lambda\right)$ with $0 \leq \lambda<1$ and $d\left(x, \psi_{t}^{\prime} x\right)=r(x, t)$ and $\psi_{t}^{\prime \prime} x$ the point $\ell\left(x, \zeta_{\rho(x, t)+\lambda(x)}\left(\psi_{t} x\right) ; \lambda\right)$ with $0<\lambda \leq 1$ and $d\left(x, \psi_{t}^{\prime \prime} x\right)=r(x, t)$. Call $x^{\prime}$ the point on $[x, \mu(x)]$ with $d\left(x, x^{\prime}\right)=r(x, t)$ and let $\delta(x, t):=d\left(\psi_{t} x, \psi_{t}^{\prime} x\right), \delta^{\prime}(x, t):=d\left(\psi_{t}^{\prime} x, \psi_{t}^{\prime \prime} x\right), \delta^{\prime \prime}(x, t):=d\left(\psi_{t}^{\prime \prime} x, \zeta_{\rho(x, t)+\lambda(x)}\left(\psi_{t} x\right)\right)$, and $D(x, t):=\delta(x, t)+\delta^{\prime}(x, t)+\delta^{\prime \prime}(x, t)$. Then we join $\psi_{t} x$ linearly to $\psi_{t}^{\prime} x$ as $\Lambda$ increases from 0 to $\delta(x, t) / D(x, t)$. Then we use the $\Lambda$-interval $[\delta(x, t) / D(x, t),(\delta(x, t)$ $\left.\left.+\delta^{\prime}(x, t)\right) / D(x, t)\right]$ to join $\psi_{t}^{\prime} x$ to $\psi_{t}^{\prime \prime} x$ on a great circle, where we define a path on a great circle as follows: If $x^{\prime}, y, z$ belong to the same simplex as $x$ or to an adjacent one, if $r:=d\left(x, x^{\prime}\right)=d(x, y)=d(x, z)$ and if $y \neq x^{\prime}$ and $z \neq x^{\prime}$, we denote, for $0 \leq \lambda \leq 1$, by $\operatorname{gc}_{x, x^{\prime}}(y, z ; \lambda)$ the point $w$ with $d(x, w)=r$ such that the broken line from $x^{\prime}$ to $w$ meets the broken line from $y$ to $z$ in $\ell(y, z ; \lambda)$. Finally, we use the $\Lambda$-interval $\left[\left(\delta(x, t)+\delta^{\prime}(x, t)\right) / D(x, t), 1\right]$ to join $\psi_{t}^{\prime \prime} x$ linearly to $\zeta_{\rho(x, t)+\lambda(x)}\left(\psi_{t} x\right)$. Formally, and more precisely:

$$
f_{\alpha}(x, t):=\left\{\begin{array}{l}
\ell\left(\psi_{t} x, \psi_{t}^{\prime} x ; \frac{D(x, t)}{\delta(x, t)} \Lambda(x, t)\right), \quad \text { if } 0 \leq \Lambda(x, t) \leq \frac{\delta(x, t)}{D(x, t)}, \\
\operatorname{gc}_{x, x^{\prime}}\left(\psi_{t}^{\prime} x, \psi_{t}^{\prime \prime} x ; \frac{D(x, t)}{\delta^{\prime}(x, t)} \Lambda(x, t)-\frac{\delta(x, t)}{\delta^{\prime}(x, t)}\right), \\
\quad \text { if } \frac{\delta(x, t)}{D(x, t)} \leq \Lambda(x, t) \leq \frac{\delta(x, t)+\delta^{\prime}(x, t)}{D(x, t)}, \\
\ell\left(\psi_{t}^{\prime \prime} x, \zeta_{\rho(x, t)+\lambda(x)}\left(\psi_{t} x\right) ; \frac{D(x, t)}{\delta^{\prime \prime}(x, t)} \Lambda(x, t)-\frac{\delta(x, t)+\delta^{\prime}(x, t)}{\delta^{\prime \prime}(x, t)}\right), \\
\quad \text { if } \frac{\delta(x, t)+\delta^{\prime}(x, t)}{D(x, t)} \leq \Lambda(x, t) \leq 1 .
\end{array}\right.
$$

If, finally, $r(x, t) / 2 \leq \theta(x, t) \leq r(x, t)$, we define $f_{\alpha}(x, t)$ in the following way: Call

$$
\lambda_{-}:=\min \left\{\lambda>0 \mid d\left(x, \ell\left(\psi_{t} x, \zeta_{\rho(x, t)+\lambda(x)}\left(\psi_{t} x\right) ; \lambda\right)\right)=r(x)\right\}
$$

and

$$
\lambda_{+}:=\max \left\{\lambda>0 \mid d\left(x, \ell\left(\psi_{t} x, \zeta_{\rho(x, t)+\lambda(x)}\left(\psi_{t} x\right) ; \lambda\right)\right)=r(x)\right\},
$$

let $\bar{\psi}_{t} x:=\ell\left(\psi_{t} x, \zeta_{\rho(x, t)+\lambda(x)}\left(\psi_{t} x\right) ; \lambda_{-}\right)$and $\overline{\bar{\psi}}_{t} x:=\ell\left(\psi_{t} x, \zeta_{\rho(x, t)+\lambda(x)}\left(\psi_{t} x\right) ; \lambda_{+}\right)$and let $\hat{\psi}_{t} x:=\mathrm{gc}_{x, x^{\prime}}\left(\psi_{t}^{\prime} x, \bar{\psi}_{t} x ; \frac{2 \theta(x, t)}{r(x, t)}-1\right)$ and $\hat{\hat{\psi}}_{t} x:=\mathrm{gc}_{x, x^{\prime}}\left(\psi_{t}^{\prime \prime} x, \overline{\bar{\psi}}_{t} x, ; \frac{2 \theta(x, t)}{r(x, t)}-1\right)$. Then we let

$$
\delta(x, t):=d\left(\psi_{t} x, \hat{\psi}_{t} x\right), \delta^{\prime}(x, t):=d\left(\hat{\psi}_{t} x, \hat{\hat{\psi}}_{t} x\right), \delta^{\prime \prime}(x, t):=d\left(\hat{\hat{\psi}}_{t} x, \zeta_{\rho(x, t)+\lambda(x)}\left(\psi_{t} x\right)\right),
$$

and $D(x, t):=\delta(x, t)+\delta^{\prime}(x, t)+\delta^{\prime \prime}(x, t)$ and define $f_{\alpha}(x, t)$ by the same formula as above where we replace $\psi_{t}^{\prime} x$ by $\hat{\psi}_{t} x$ and $\psi_{t}^{\prime \prime} x$ by $\hat{\psi}_{t} x$. Obviously, we will then have $f_{\alpha}(x, t) \neq x$ in this situation. If $\rho(x, t)=\delta_{0} / 16$, we have that $\Lambda(x, t)=1$, and hence $f_{\alpha}(x, t)=\zeta_{\rho(x, t)+\lambda(x)}\left(\psi_{t} x\right)$; so the definition agrees with the one given in 2 ). If $\rho(x, t)=\delta_{0} / 8$, we have that $\Lambda(x, t)=0$, so $f_{\alpha}(x, t)=\psi_{t} x$; so our definition agrees with the one in 1$)$. If $\theta(x, t)=r(x, t) / 2$, we have that $\hat{\psi}_{t} x=\psi_{t}^{\prime} x$ and $\hat{\hat{\psi}}_{t} x=\psi_{t}^{\prime \prime} x$; so the definitions for $0 \leq \theta(x, t) \leq r(x, t) / 2$ and $r(x, t) / 2 \leq \theta(x, t) \leq$ $r(x, t)$ agree in this case. If $\theta(x, t)=r(x, t)$, we have that $\lambda_{-}=\lambda_{+}=: \lambda_{0}$, and so $\hat{\psi}_{t} x=\bar{\psi}_{t} x=\overline{\bar{\psi}}_{t} x=\hat{\hat{\psi}}_{t} x=\ell\left(\psi_{t} x, \zeta_{\rho(x, t)+\lambda(x)}\left(\psi_{t} x\right) ; \lambda_{0}\right)$. But then $\delta^{\prime}(x, t)=0$, and $f_{\alpha}(x, t)=\ell\left(\psi_{t} x, \zeta_{\rho(x, t)+\lambda(x)}\left(\psi_{t} x\right) ; \Lambda(x ; t)\right)$. Moreover, as $d\left(x, A_{0}\right)$ tends to 0 , we have that $r(x, t)$ tends to 0 which shows that we have defined $f_{\alpha}$ in a continuous manner.

5. Finally, in order to complete the construction of $f_{\alpha}$, we extend $f_{\alpha}$ in an arbitrary continuous way over the remaining simplices — for example, we could use 
the same procedure as in 4) if we replace great circles by straight lines if the points in question are collinear. Of course, we will then not have that $f_{\alpha}(x, t) \neq x$ but this wasn't required in the proof.

There still remains a minor point: We still have to show that we may choose a convenient approximation so as to fulfill the additional requirements in part 3.4 in the proof of the theorem. So we assume the situation of 3.4 and we retain the notation of that section. We will sketch the construction of an approximation $f_{\alpha \beta}$ to the map $(x, \tau, t) \mapsto h\left(p_{\alpha} \phi_{t} i_{\alpha \cup \beta} h(x, \tau), \tau\right)=: \psi_{t}(x, \tau)$ for $(x, t) \in \operatorname{cl} j_{\alpha}^{-1}(U)$ and $0 \leq \tau \leq 1$. We choose again a neighbourhood $D$ of the subcomplex of $N_{\alpha \cup \beta}$ containing all vertices corresponding to elements of $\alpha^{*} \cup \beta^{*}$ and we choose a function $\zeta$ as in the supplementing remark after the lemma such that $\zeta_{s}(h(x, \tau)) \in N_{\tau}$ if $x \in N_{\alpha} \backslash D, 0 \leq \tau \leq 1$, and $\delta_{0} / 16 \leq s \leq 3 \delta_{0} / 8$. We choose the numbering of the distinguished orbits as in Part 4 , and we define $\delta(x), \delta_{0}$, and $\rho(x, t)$ for the mapping $\psi_{t}$ just defined as above. If $\rho(x, t) \geq \delta_{0} / 8$, we let $f_{\alpha \beta}(x, \tau, t)=\psi_{t}(x, \tau)$. Also, if $x \in \gamma_{j}^{\prime}$ for $j \in\{1, \ldots, r+u\}$ and $t$ a period of $x$, we let $f_{\alpha \beta}(x, \tau, t)=\psi_{t}(x, \tau)$. For $j \in\{r+u+1, \ldots, s\}$ and $x \in \gamma_{j}^{\prime}$ we let $f_{\alpha \beta}(x, 0, t)=\psi_{t}(x, 0)$. For the remaining points we then proceed exactly as in the proof of the existence of a convenient approximation arranging things in such a way that $f_{\alpha \beta}(h(x, \tau), t) \in N_{\tau}$. This is possible since $\psi_{t}(x, \tau) \in N_{\tau}$ and $\zeta_{s}(h(x, \tau)) \in N_{\tau}$ if $x \in N_{\alpha} \backslash D, 0 \leq \tau \leq 1$, and $\delta_{0} / 16 \leq s \leq 3 \delta_{0} / 8$. Whenever $x$ is required to be in a simplex $\sigma$, we replace this by the condition that $h(x, \tau)$ be in a simplex $\sigma$ of $N_{\tau}$ (by giving $N_{\tau}$ the structure of $N_{\alpha}$ for $\left.0 \leq \tau<1\right)$. Moreover, in the construction of the convenient approximation we had to choose a nearest point $\mu(x)$ in $A_{0}$. If we insist on choosing $\mu(x) \in N_{\tau}$ for $x \in N_{\tau}$ the construction of a convenient approximation will consist in joining points in $N_{\tau}$ by straight line segments or great circles, so that $f_{\alpha \beta}(h(x, \tau)) \in N_{\tau}$ as was required in 3.4. This finishes the construction of a convenient approximation.

In concluding, I would like to express my gratitude to the Jagiellonian University of Cracow and in particular to Professors Mrozek, Pelczar and Srzednicki for their kind hospitality when I started working on this problem.

\section{REFERENCES}

[CMP] S.N. Chow and J. Mallet-Paret, The Fuller index and global Hopf bifurcation, J. Differential Equations 39 (1978), 66 - 84. MR 58:11665

[DT] N. Dancer and J.F. Toland, Degree theory for orbits of prescribed period of flows with a first integral, Proc. London Math. Soc. 60 (1990), 549 - 580. MR 91a:58152

[Du] J. Dugundji, Topology, Allyn and Bacon, Boston, 1978. MR 57:17581

[F1] C.C. Fenske, Periodic orbits of semiflows - Local indices and sections, Selected Topics in Operations Research and Mathematical Economics. Proceedings, Karlsruhe 1984 (G. Hammer and D. Pallaschke, eds.), Lecture Notes in Economics and Mathematical Systems 226, Springer, Berlin/Heidelberg/New York, 1984, pp. 348-360. MR 86i:54035

[F2] - A simple-minded approach to the index of periodic orbits, J. Math. Anal. Appl. 129 (1988), 517-532. MR 89g:54098

[F3] _ An index for periodic orbits of functional differential equations, Math. Ann. 285 (1989), 381-392. MR 90m:34135

[Fu] F.B. Fuller, An index of fixed point type for periodic orbits, Amer. J. Math. 89 (1967), 133-148. MR 35:497

[GN] R. Geoghegan and A. Nicas, Trace and torsion in the theory of flows, Topology 33 (1994), 683-719. MR 95h:55002

[G] J. Girolo, Approximating compact sets in normed spaces, Pacific J. Math. 98 (1982), 81-89. MR 83c: 54046

$[\mathrm{H}] \quad$ S.-T. Hu, Theory of retracts, Wayne State University Press, Detroit, 1965. MR 31:6202 
[M] W.S. Massey, Homology and Cohomology Theory, Marcel Dekker, New York/Basel, 1978. MR 58:7594

[P1] A.J.B. Potter, On a generalization of the Fuller index, Nonlinear functional analysis and its applications (Felix Browder, ed.), Proc. Symp. Pure Math. 45, Part 2, AMS, Providence, 1986, pp. 283-286. MR 87g:47112

[P2] Approximation methods and the generalised Fuller index for semi-flows in Banach spaces, Proc. Edinburgh Math. Soc. 29 (1986), 299-308. MR 87m:47121

[Sr1] R. Srzednicki, Periodic orbit indices, Fund. Math 135 (1990), 147-173. MR 91m:54054

[Sr2] , Topological invariants and detection of periodic orbits, J. Differential Equations 111 (1994), 283-298. MR 95g:58186

Mathematisches Institut der Justus-Liebig, Universität Arndtstrasse, 2 D-35392 Giessen, Germany

E-mail address: christian.fenske@math.uni-giessen.d400.de 